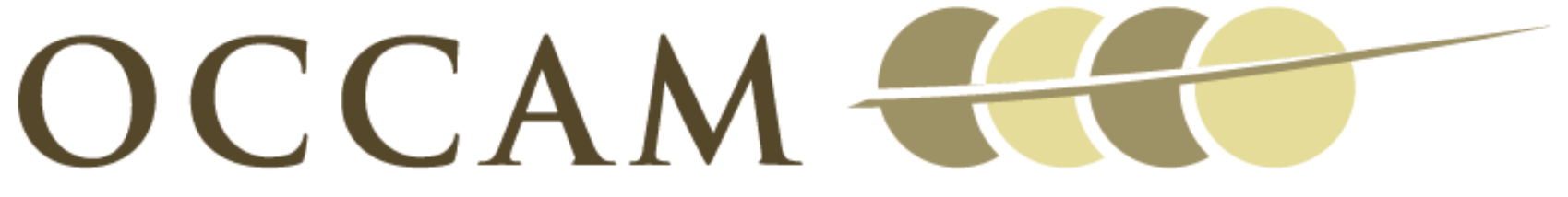

OXFORD CENTRE FOR COLLABORATIVE APPLIED MATHEMATICS

Report Number 09/24

The effect of polar lipids on tear film dynamics by

E. Aydemir, C.J.W. Breward and T. P. Witelski

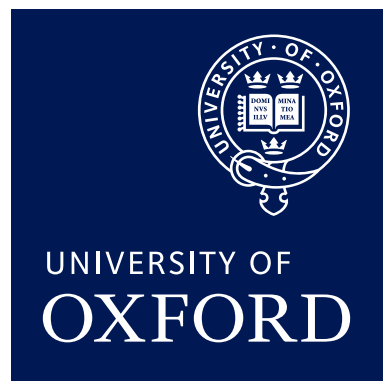

Oxford Centre for Collaborative Applied Mathematics Mathematical Institute 24 - 29 St Giles'

Oxford

OX1 3LB

England 



\title{
The effect of polar lipids on tear film dynamics
}

\author{
E. Aydemir ${ }^{a}$, C. J. W. Breward ${ }^{a, *}$ and T. P. Witelski ${ }^{b}$
}

July 27, 2009

a Mathematical Institute, University of Oxford, 24-29 St Giles', Oxford, OX1 3LB, United Kingdom

${ }^{b}$ Mathematics Department, Duke University, Box 90320, Durham, NC 27708-0320, United States

\begin{abstract}
In this paper we present a mathematical model describing the effect of polar lipids on the evolution of a precorneal tear film, with the aim of explaining the interesting experimentally observed phenomenon that the tear film continues to move upwards even after the upper eyelid has become stationary. The polar lipid is an insoluble surface species that locally alters the surface tension of the tear film. In the lubrication limit, the model reduces to two coupled nonlinear partial differential equations for the film thickness and the concentration of lipid. We solve the system numerically and observe that the presence of the lipid causes an increase in flow of liquid up the eye. We further exploit the size of the parameters in the problem to explain the initial evolution of the system.
\end{abstract}

Keywords Tear Films; Lipids; Blinking; Fluid Mechanics; Lubrication Theory.

\section{Introduction}

The dynamics of tear films are of particular interest to ophthalmologists due to the prevalence of dry eye [16], a multifactorial disease of the tears and ocular surface that results in symptoms of discomfort, visual disturbance, and tear film instability with the potential to damage the ocular surface. It is accompanied by increased osmolarity of the tear film and inflammation of the ocular surface. In severe dry eye, the tear film can thin to rupture over short periods of time; frequent blinking is vital to reform and re-establish the tear film and alleviate these symptoms.

The functions of the tear film are: to provide an optically smooth surface, to protect against the bacterial contamination of the exposed sclera, and to lubricate the gap between the eyelids and the cornea of the eye (Holly [8]). The tear film comprises three layers: an aqueous layer sandwiched between a bi-component oily lipid surface layer and a mucous coating that lies on the corneal epithelium. The aqueous component is produced by the lacrimal glands, fills the sacks under the upper and lower eyelids and accounts for the majority of the tear fluid. The lipid layer is secreted from the meibomian glands of the eyelid, decreases the surface tension of the tear film and reduces evaporation of the aqueous layer. The lipid layer contains both polar and nonpolar lipids, which are present in the ratio of about 1:10. The polar lipids reside at the aqueous interface, while the

\footnotetext{
${ }^{*}$ Corresponding author. Email addresses: breward@maths.ox.ac.uk, witelski@math.duke.edu
} 
1.

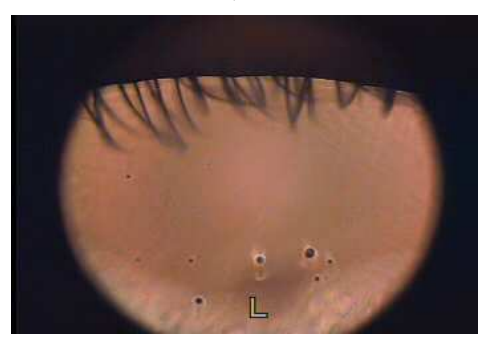

3.

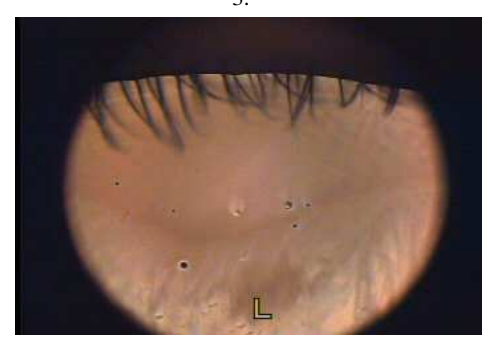

2.

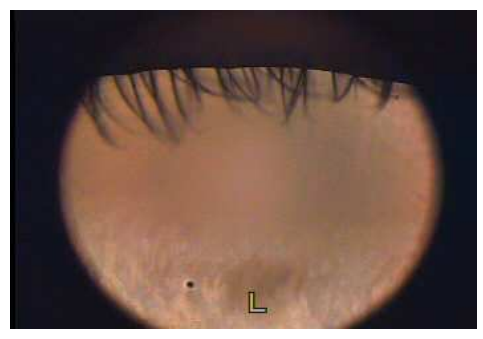

4.

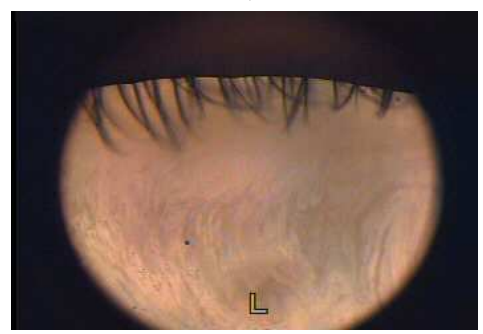

Figure 1: The above sequence of fluorescein images of a human left eye, taken after a blink, shows the upward movement of the lipid layer. Image 1 was taken immediately after the upstroke motion of the upper eyelid, followed closely by 2,3 and then 4 . (Image kindly supplied by A. Bron and J. Tiffany, courtesy of N. Yokoi.)

non-polar lipids reside above. A study by Nagyová and Tiffany [21] found almost no dissolved lipid in tears extracted from human eyes, indicating that both components of the lipid layer are insoluble. The mucus layer is secreted from cells which are situated in the conjunctival epithelium. McCulley and Shine [19] claim that data supports the case for a mucus layer that blends with the aqueous layer without any interfacial tension between the two. Holly [8] argues that, physico-chemically, the mucus layer should be considered part of the solid epithelium.

Blinking is the mechanism by which the tear film gets redistributed over the surface of the eye. Blinking is predominately attributed to the movement of the upper eyelid, which sweeps down to meet the nearly stationary lower eyelid, and then sweeps upwards and redistributes the tear film over the eye surface. The deposited tear film has menisci at each of the eyelids, joined by a thin layer that covers the eye surface. The opening phase takes approximately a third of a second, and then the eye remains open for "the interblink", a further period of five to ten seconds, before the process is repeated, normally involuntarily. Some people only undergo partial blinks, where the upper eyelid only travels part of the way over the eye surface before returning upwards. The tear film provides a separation between the cornea and the lids, as well as between the upper and lower eyelids when the eyelid is in the closed position, reducing the surface-to-surface friction during the blinking process. During a blink, there is exchange of liquid between the exposed tear film and the fluid under the upper eyelid. However, experiments by MacDonald and Maurice [17], involving the deposition of a small drop of fluorescein solution at the bottom of the the lower fornix, found that the average time for the fluorescein to appear in the tear film was around four minutes. This indicates that there is no appreciable transfer of liquid from under the lower eyelid on the timescale of an individual blink.

During the interblink, the tear film thins due to a combination of evaporation and capillary 
suction into the menisci. Further, the lipid layer on the tear film is still observed to move upward after the eyelids have stopped moving, as can be seen in Figure 1. Here, it is the nonpolar lipid that can be seen as the lighter coloured layer advancing up the stills. In King-Smith et al [14], the upward motion of the lipid is also noted. They examine the liquid motion caused by capillary suction into the menisci and conclude that it is not large enough to account for the thinning rate inferred from their data.

Tear films are have previously been modelled as a thin film of Newtonian liquid moving over a solid surface. The aspect ratio of the problem is used to reduce the Navier-Stokes equations to the equations of lubrication theory $[2,3,7,11,12,26]$.

The upward drift of the tear film after a blink is studied by Berger and Corrsin [2]. By examining the movement of particles on the tear film they conclude that the upper tear film thickens for one second immediately after the upper eyelid has stopped moving, while the lower tear film thins simultaneously. The surface tension is assumed to be a known function of the lipid concentration. Utilising a small displacement approximation, they solve the resulting system of equations for the film thickness and the lipid concentration in the case when the eye was open. They calculate particle trajectories and compare these with experimental data, finding good agreement.

The coating model presented by Wong et al[26] is the simplest model to capture the dynamic evolution of the tear film thickness. The model includes capillary and viscous forces and assumes that the surface velocity is equal to the upper eyelid velocity so that the lipid is dragged along with the movement of the upper eyelid. By decomposing the tear film (at the upper eyelid) into a meniscus, deposition layer and central region, they predict a power law behaviour for the film thickness in the central region as a function of the capillary number.

Braun and Fitt [3] investigate the influence of gravity and evaporation on an established tear film. They solve for the temperature in the film and relate the rate of evaporation to the surface temperature. They argue that evaporation plays a small role during the early stages of the thinning process but that it plays a more significant role once the film has thinned to approximately $1 \mu \mathrm{m}$. They conclude that evaporation always shortens the lifetime of the tear film and that gravity dominates the evolution away from the menisci and causes the region near the upper lid to thin faster than the region near the lower eyelid.

Jones et al[12] extend the model used by Braun and Fitt [3] to include lipids in a rudimentary way. Their model includes capillary, viscous and gravity forces and assumes that the lipid generates a surface velocity, $u_{s}$, given by $u_{s}=(x / L) \dot{L}$, where $x$ is the distance along the film, and $L$ is the position of the moving upper eyelid. This is analogous to a uniform stretching of a "membrane" of lipid on the surface. They allow for the transfer of liquid from underneath the eyelids (assuming that there is a liquid layer of uniform thickness under the upper eyelid). They conclude that the supply of liquid from under the eyelids is crucial to establish a tear film that covers the whole of the open eye: it's not possible to fully deposit a tear film without this flux. The presence of the lipid assisted with the deposition of the tear film, but the film was again not fully deposited when there was no flux under the eyelids.

In Jones et al[11], the model from [3] was extended to track the concentration of the lipid. The coupled equations for the film thickness and the lipid concentration were first solved on a stationary domain with a nonuniform initial concentration of lipid to assess spreading, and then solved on the moving domain. In the dynamic case they include a flux from under the upper eyelid, and they reaffirm that the tear film cannot be fully deposited without this flux. They highlight that the evolution of the lipid layer can have a strong influence on the height of the tear film. 
In Braun and King-Smith [4], the coupled model for the thickness of the film and the concentration of lipids is extended to include "slip" at the eye surface. The model is solved in the stress-free surface case and in the large Marangoni number limit, where the model reduces to that of [12]. Sinusoidal motion of the eyelids is considered and both blinks and half-blinks are examined. They conclude that the model reproduces tear film profiles measured in vivo for half blinks. This work is extended in Heryudono et al[7] to incorporate a more realistic eyelid velocity and flux under both eyelids to take account of supply from the lacrimal gland and drainage due to the puncta. The model is solved in the two cases described in [4] and compared with the solution of previous models and with experiments.

In this article, we formulate and study a model for the evolution of the thickness of the tear film and the concentration of polar lipids ${ }^{1}$ during the blinking process, with the aim of understanding how the presence of polar lipids influences the liquid flow. In Section 2, we present some experimental data on the motion of the eyelids and fit the data to a model. In Section 3 we formulate a model for the aqueous and lipid components of the system. This model is nondimensionalised and the size of the nondimensional parameters discussed. Utilising the lubrication approximation, we reduce the equations and boundary conditions to a thin-film limit, and the resulting model comprises two coupled nonlinear PDEs for the thickness of the tear film and the concentration of the nonpolar lipid. In section 5 we present numerical simulations of the reduced system and discuss their behaviour as various critical nondimensional parameter groups are varied. In section 6 , the asymptotic behaviour of the model at the start of the blink is examined and compared with the numerical solutions. Finally, we draw together our conclusions and suggest possible future avenues for investigation.

\section{Experimental data on the motion of the eyelids}

To determine the typical position and velocity of the upper eyelid at a particular time we will use the measurements presented by Wong et al [26]. However, we use a different functional form than the one employed by Jones et al[12], because their form for the velocity dictates that it takes an infinite amount of time for the eyelid to come to rest. In this paper, we study the fluid motion as the eyelid opens and after it becomes stationary for a situation where the eye is held open for an sustained length of time.

The strong initial acceleration motivates us to use the form

$$
U(t)= \begin{cases}U_{0}\left(\lambda \sqrt{t / \tau} e^{-t / \tau}-t / \tau\right) & 0 \leq t \leq t^{*} \\ 0 & t>t^{*}\end{cases}
$$

where $U_{0}, \lambda$, and $\tau$ are found by fitting (1) to Wong's data [26] and take the approximate values

$$
U_{0}=0.0163 \mathrm{~m} / \mathrm{s}, \quad \lambda=11.6, \quad \tau=0.0865 \mathrm{~s},
$$

see Figure 2. The constant $t^{*}$ is the time at which the velocity becomes zero, found by solving $\lambda^{2} \tau e^{-2 t^{*} / \tau}=t^{*}$, yielding $t^{*} \approx 0.180 \mathrm{~s}$.

The position, $L(t)$, of the upper eyelid is defined by $U(t)=\dot{L}(t)$, so that

$$
L(t)=L_{c l}+U_{0} \tau\left(-\frac{1}{2}\left[\frac{t}{\tau}\right]^{2}+\lambda\left[\frac{\sqrt{\pi}}{2} \operatorname{erf}\left(\sqrt{\frac{t}{\tau}}\right)-\sqrt{\frac{t}{\tau}} e^{-t / \tau}\right]\right)
$$

\footnotetext{
${ }^{1}$ We will neglect the non-polar lipid in this paper.
} 
(a)

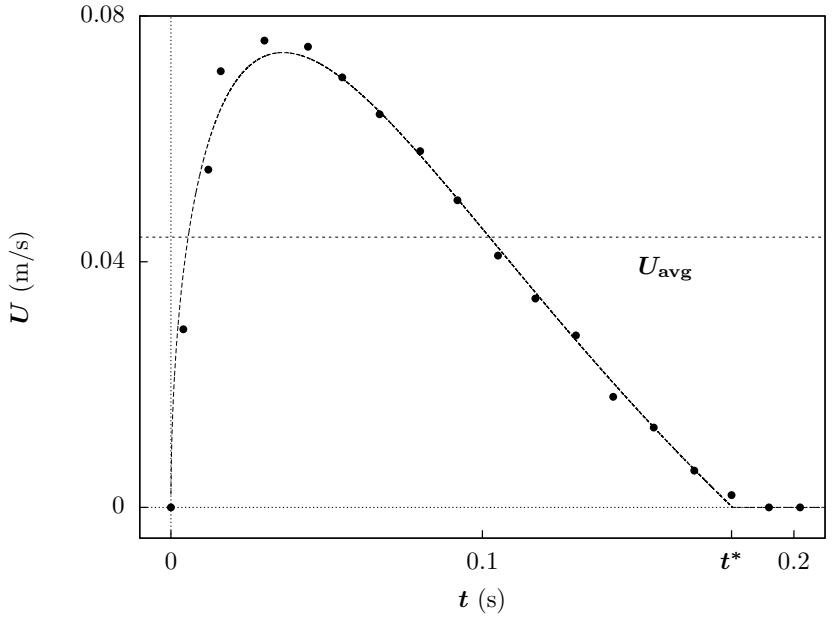

(b)

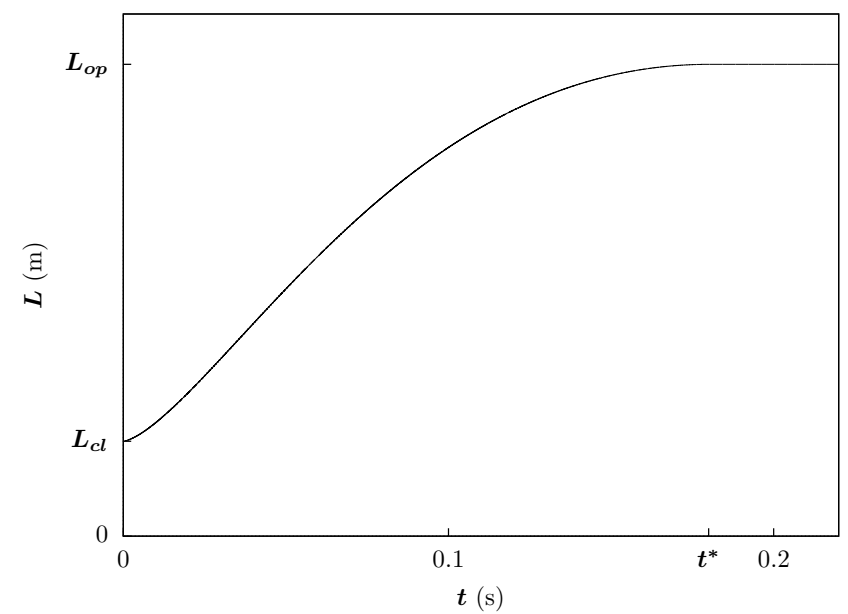

Figure 2: (a) The speed of the upper eyelid during the opening phase of a blink, $U(t)$ (1) with parameters (2) data points extracted from Wong [26] fit to $(1,2)$. (b) Corresponding separation length $L(t)$ given by $(3)$.

where $L_{c l}$ is the effective position of the closed upper eyelid at $t=0$. For $T=0.180 \mathrm{~s}$ we use (3) to determine that $L_{c l}=0.002 \mathrm{~m}$ is consistent with a distance between the eyelids, $L_{o p}$ of $0.01 \mathrm{~m}$ when the eye is fully open. The finite separation for the closed eyelids accounts for volume of the tear fluid that will spread out over the eve when the eyelids separate. Using these values, we are able to calculate an average velocity for the opening phase, which is found by calculating

$$
U_{a v}=\frac{1}{T} \int_{0}^{T} U(t) d t,=0.044 \mathrm{~m} / \mathrm{s} .
$$

These are approximate values and, in particular, from the data we are only able to determine the distance that the upper eyelid had moved (i.e. $L_{o p}-L_{c l}$ ) rather than $L_{o p}$ and $L_{c l}$ independently. ${ }^{2}$

\section{Mathematical model for the evolution of the tear film}

In this paper we are primarily interested in assessing whether the Marangoni traction exerted by the polar lipid can result in the upward movement of the tear film shown in Figure 1. The liquid of the tear film is constrained to lie on the surface of the eyeball and between the eyelids. We assume that the tear film thickness is always thinner than its length ${ }^{3}$, and we will exploit the slenderness of the geometry in order to simplify the problem, see Figure 3. We neglect the presence of the non-polar lipid.

We treat the aqueous and mucus layers as one continuous layer of a Newtonian liquid. The eyeball will be assumed to remain stationary during the blink. Our starting point is the two-

\footnotetext{
${ }^{2}$ Of course, another choice for the distance between the eyelids when open would have resulted in a different value for $L_{c l}$. We proceed on the assumption that this will not significantly alter our results, and that, in future, a more complete data set may become available.

${ }^{3}$ We note that this may not be true when the eyelids are very close together, but we proceed anyway.
} 


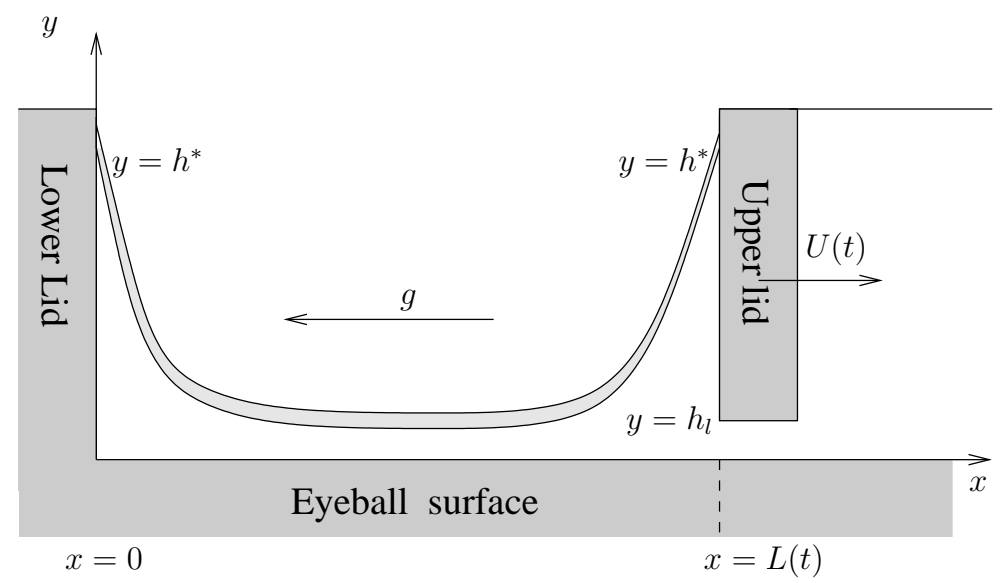

Figure 3: Schematic of the tear film problem. The shaded layer on the tear film surface indicates the insoluble lipid.

dimensional Navier-Stokes equations [22],

$$
\begin{gathered}
\nabla \cdot \mathbf{u}=0 \\
\rho\left(\mathbf{u}_{t}+(\mathbf{u} \cdot \nabla) \mathbf{u}\right)=-\nabla p+\mu \nabla^{2} \mathbf{u}-\rho g \mathbf{i},
\end{gathered}
$$

where, in Cartesian coordinates, the fluid velocity is given by $\mathbf{u}=(u(x, y, t), v(x, y, t))$, with pressure $p$, density $\rho$, viscosity $\mu$ and gravity $g$ (acting in the $-\mathbf{i}$ direction). These equations represent conservation of mass and momentum, respectively.

The radius of curvature of the eyeball is similar to the distance between the eyelids when the eye is open, and so, strictly speaking, we should work in curvilinear coordinates and consider the effect of the (constant) curvature of the eyeball on the evolution of the film. However, to focus the influences of the forces, we neglect geometrical effects, by assuming that the eyeball surface is flat. The corneal epithelium is assumed to be a flat impermeable solid surface, and so we assume no slip and no flux conditions, given by the boundary conditions

$$
u=0, \quad v=0, \quad \text { at } y=0 .
$$

The motion of the tear film free-surface, $y=h(x, t)$, is given by the kinematic boundary condition,

$$
v=h_{t}+u h_{x}-E \sqrt{1+h_{x}^{2}}, \quad \text { at } y=h,
$$

where $E$ is the mass transfer through the surface due to evaporation. We denote the stress in the fluid by $\tau=-p \mathbf{I}+\mu\left[\nabla \mathbf{u}+\nabla \mathbf{u}^{T}\right]$ and let $\mathbf{n}$ be the unit normal to the surface and $\mathbf{t}$ be the unit tangent. At the air-liquid interface, the normal stress is balanced by the surface tension,

$$
\mathbf{n} \cdot(\tau \cdot \mathbf{n})=\gamma \kappa,
$$

where $\kappa$ is curvature of the interface and $\gamma$ is the surface tension (which depends on the local concentration of the lipid). Similarly, the tangential stress is balanced by the surface tension gradient (or Marangoni stress)

$$
\mathbf{t} \cdot(\tau \cdot \mathbf{n})=\mathbf{t} \cdot \nabla_{s} \gamma
$$


where $\nabla_{s}$ is the surface gradient.

For the free surface $y=h(x, t)$ these stress balances reduce to

$$
\begin{aligned}
p_{0}-p+2 \mu \frac{v_{y}+u_{x} h_{x}^{2}-h_{x}\left(u_{y}+v_{x}\right)}{1+h_{x}^{2}} & =\gamma \frac{h_{x x}}{\left(1+h_{x}^{2}\right)^{3 / 2}} \\
\mu \frac{2\left(v_{y}-u_{x}\right) h_{x}+\left(u_{y}+v_{x}\right)\left(1-h_{x}^{2}\right)}{1+h_{x}^{2}} & =\frac{\gamma_{x}}{\sqrt{1+h_{x}^{2}}},
\end{aligned}
$$

where $p_{0}$ is the atmospheric pressure.

We assume that the polar lipid layer acts like an insoluble surfactant, that is, it is present only on the film surface and absent in the bulk of the liquid. The dynamics of such a species is driven by surface convection and diffusion as described in the mass transport equation (see [24])

$$
\Gamma_{t}+\frac{\partial}{\partial s}\left(u_{s} \Gamma\right)-D_{s} \frac{\partial^{2} \Gamma}{\partial s^{2}}+\kappa u_{n} \Gamma=0,
$$

where $u_{s}=\left.\mathbf{u} \cdot \mathbf{t}\right|_{y=h(x, t)}$ and $u_{n}=\left.\mathbf{u} \cdot \mathbf{n}\right|_{y=h(x, t)}$ are the surface velocity and velocity normal to the surface, $D_{s}$ is the surface diffusion coefficient and we have written out the derivatives with respect to arc length to avoid confusion with the use of subscript $s$ to denote the surface velocity.

In order to complete the model we have to relate the surface tension of the liquid to the concentration of the lipid. We assume that the lipid is present in dilute concentration, which allows us to adopt a linearised version of the model derived by Burdon [6],

$$
\gamma=\gamma_{0}-R T \Gamma
$$

where $R$ is the universal gas constant $R=8.31 \mathrm{~J} / \mathrm{mol} \mathrm{K}$ and $T(\approx 310 \mathrm{~K})$ is the absolute temperature. This model was also employed by Berger and Corrsin [2].

\subsection{Boundary conditions at eyelids}

The meibomian glands are situated at the division between the hydrophilic and hydrophobic region of the lid margins, known as the muco-cutaneous junction. The aqueous component of the tear liquid cannot pass onto the hydrophobic cells and the liquid becomes pinned along this line, where the fluid film is able to assume any contact angle. Hence we prescribe the height of the tear film at either end of the region,

$$
h(0, t)=h(L, t)=h^{*} .
$$

We assume that no liquid escapes under the lower eyelid and we assume that the upper eyelid delivers a flux $Q$ of liquid into the tear film as the eye opens. The simplest assumption for the flux supplied by the upper eyelid is that the gap $h_{l}$ between the bottom of the eyelid and the eyeball is fixed, giving $Q=h_{l} \dot{L}$. We proceed under this assumption, noting that we expect that, in practice, the height of this gap will vary as the speed of the eyelid changes. Additionally, we suppose that no lipid is supplied through either eyelid.

At the lower eyelid the boundary conditions read

$$
\bar{u}=0, \quad u_{s}=0, \quad \text { at } \quad x=0,
$$


where $\bar{u}$ is the average velocity of the liquid in the tear film, while at the upper (moving) lid we must impose

$$
\bar{u}=\left(1-\frac{h_{l}}{h^{*}}\right) \dot{L}, \quad u_{s}=\dot{L}, \quad \text { at } \quad x=L,
$$

where $L$ is given by (3).

\subsection{Initial conditions}

We suppose that, initially, the eyelids are almost closed, with distance between them as determined in section 2. Further, we suppose that the fluid layer has uniform thickness $h^{*}$ and that the amount of lipid is known and evenly distributed with concentration $\Gamma^{*}$. Thus we write,

$$
L=L_{c l}, \quad h=h^{*}, \quad \Gamma=\Gamma^{*} \quad \text { at } \quad t=0 .
$$

\section{Nondimensionalisation}

We assume that it is always true that the typical distance between the eyelids is much larger than the height of the pinned meniscus, $h^{*}$ and that $h^{*}$ is much larger than the thickness $h_{0}$ of the majority of the film. We define an aspect ratio based on the thickness of the majority of the film, $\epsilon=h_{0} / L_{o p} \ll 1$. We anticipate that, because the film thickness near the eyelids is significantly larger than in the majority of the film, there may be "boundary layers" close to the eyelids in which different scalings may be more appropriate.

We proceed by nondimensionalising (3), (5a), (5b), (6b), (8) and (9) using the following scalings,

$$
\begin{aligned}
& x=L_{o p} x^{\prime}, \quad y=\epsilon L_{o p} y^{\prime}, \quad L=L_{o p} L^{\prime}, \quad t=\frac{L_{o p}}{U} t^{\prime}, \\
& u=U u^{\prime}, \quad v=\epsilon U v^{\prime}, \quad p=p_{0}+\frac{\mu U}{L_{o p} \epsilon^{2}} p^{\prime}, \quad h=\epsilon L_{o p} h^{\prime} \\
& \Gamma=\Gamma^{*} \Gamma^{\prime}, \quad E=E_{0} E^{\prime} .
\end{aligned}
$$

Since we assume that the concentration of lipid is small, variations in $\gamma$ are assumed to be small, i.e. gradients are given by $\gamma_{x}=R T \Gamma_{x}$ but the concentration is approximated by $\gamma \sim \gamma_{0}$. We note that we have not yet specified the velocity scaling $U$ that we wish to employ: we do this later in section 4.1.

Immediately dropping the primes, the nondimensional model reads

$$
\begin{gathered}
u_{x}+v_{y}=0 \\
\epsilon^{2} \operatorname{Re}\left(u_{t}+u u_{x}+v u_{y}\right)=-p_{x}-\mathrm{St}+\epsilon^{2} u_{x x}+u_{y y} \\
\epsilon^{4} \operatorname{Re}\left(v_{t}+u v_{x}+v v_{y}\right)=-p_{y}+\epsilon^{4} v_{x x}+\epsilon^{2} v_{y y}
\end{gathered}
$$

where $\operatorname{Re}=\rho U L_{o p} / \mu$ is the Reynolds number, and the scaled Stokes number is St $=\rho g L_{o p}^{2} \epsilon^{2} / \mu U$. The boundary conditions at $y=h(x, t)$ become,

$$
v=h_{t}+u h_{x}-\left(1+\epsilon^{2} h_{x}^{2}\right)^{\frac{1}{2}} \operatorname{Ev} E,
$$




$$
\begin{gathered}
\left(\frac{\epsilon^{3}}{\mathrm{Ca}}-\epsilon^{3} \mathrm{Ma} \Gamma\right)\left(\frac{h_{x x}}{\left(1+\epsilon^{2} h_{x}^{2}\right)^{3 / 2}}\right)=-p+2 \epsilon^{2} \frac{v_{y}+\epsilon^{2} u_{x} h_{x}^{2}-h_{x}\left(u_{y}+\epsilon^{2} v_{x}\right)}{1+\epsilon^{2} h_{x}^{2}} \\
-\frac{\epsilon \mathrm{Ma}_{x}}{\left(1+\epsilon^{2} h_{x}^{2}\right)^{\frac{1}{2}}}=\frac{2 \epsilon\left(v_{y}-u_{x}\right) h_{x}+\left(u_{y}+\epsilon^{2} v_{x}\right)\left(1-\epsilon^{2} h_{x}^{2}\right)}{1+\epsilon^{2} h_{x}^{2}} .
\end{gathered}
$$

where $\mathrm{Ev}=E_{0} / \epsilon U$ is the evaporation constant, $\mathrm{Ca}=\mu U / \gamma_{0}$ is the capillary number and $\mathrm{Ma}=$ $R T \Gamma^{*} / \mu U$ is the Marangoni number.

The equation for the lipid concentration (8) becomes

$$
\left(1+\epsilon^{2} h_{x}^{2}\right)^{\frac{1}{2}} \Gamma_{t}+\left(u_{s} \Gamma\right)_{x}-\frac{\Gamma_{x x}}{\operatorname{Pe}\left(1+\epsilon^{2} h_{x}^{2}\right)^{\frac{1}{2}}}-\epsilon^{2} \frac{\Gamma h_{x x}\left(v-u h_{x}\right)}{\left(1+\epsilon^{2} h_{x}^{2}\right)^{\frac{3}{2}}}=0,
$$

where the surface Peclet number is $\mathrm{Pe}=U L_{o p} / D_{s}$. The nondimensionalised boundary conditions read

$$
\begin{gathered}
\bar{u}=0, \quad u_{s}=0, \quad h=\mathcal{H}, \quad \text { at } \quad x=0, \\
\bar{u}=\left(1-\frac{\mathcal{H}_{l}}{\mathcal{H}}\right) \dot{L}, \quad u_{s}=\dot{L}, \quad h=\mathcal{H} \quad \text { at } \quad x=L,
\end{gathered}
$$

where $\mathcal{H}=h^{*} /\left(\epsilon L_{o p}\right), \mathcal{H}_{l}=h_{l} /\left(\epsilon L_{o p}\right)$ and $L$ is given by the nondimensionalised version of (3), namely

$$
L(t)=\delta+U^{*} \tau^{*}\left(-\frac{1}{2}\left[\frac{t}{\tau^{*}}\right]^{2}+\lambda\left[\frac{\sqrt{\pi}}{2} \operatorname{erf}\left(\sqrt{\frac{t}{\tau^{*}}}\right)-\sqrt{\frac{t}{\tau^{*}}} e^{-t / \tau^{*}}\right]\right),
$$

where $\delta=L_{c l} / L_{o p}, U^{*}=U_{0} / U, \tau^{*}=\tau U / L_{o p}$, and the initial conditions are given by

$$
L=\delta, \quad h=\mathcal{H}, \quad \Gamma=1 \quad \text { at } \quad t=0 .
$$

\subsection{Parameter values}

We use the following values (Jones et al: [11], Tiffany et al[25])

$$
\mu \approx 1.3 \times 10^{-3} \mathrm{Ns} / \mathrm{m}^{2}, \quad \rho \approx 1.0 \times 10^{3} \mathrm{~kg} / \mathrm{m}^{3}, \quad \gamma \approx 4.5 \times 10^{-2} \mathrm{~N} / \mathrm{m},
$$

and we further assume that

$$
L_{\text {op }}=0.01 \mathrm{~m}, \quad h_{0}=10^{-5} \mathrm{~m}, \quad h^{*}=10^{-3} \mathrm{~m}
$$

We do not have data on the size of $\Gamma^{*}$ but by analogy with other problems involving surface tension gradients (see, for example, Figure 5 in Breward et al[5]), we expect $R T \Gamma^{*}$ to be around $10^{-3} \mathrm{~N} / \mathrm{m}$ or smaller which gives

$$
\Gamma^{*} \approx 4 \times 10^{-7} \mathrm{~mol} / \mathrm{m}^{2}
$$

In determining the evaporation rate, we follow the same argument set out by Braun and Fitt [3] and Jones et al[11]. They both assume that the relationship between the rate of evaporative mass flux and the difference between the film surface temperature, $T$ and the saturation temperature of the vapour $T_{\text {sat }}$ is linear, thus $E \propto T-T_{\text {sat }}$, where, since the film is very thin, $T \approx$ constant, equal to the ocular surface temperature. The average rate of evaporation in normal eyes is given 
as $1.5 \times 10^{-5} \mathrm{~kg} / \mathrm{m}^{2} \mathrm{~s}$ (Mathers [18]). Our $E_{0}$ is a mass transfer coefficient, so we must divide the Mathers figure by the density of the liquid, yielding $E_{0}=1.5 \times 10^{-8}$. The work by Mishima [20] estimates that evaporation causes the thickness to decrease by approximately 40 per cent after several minutes, suggesting that evaporation has a negligible effect on the time scale we are considering.

The surface diffusivity $D_{s}$ for ocular lipids is unknown and we employ the estimate made by Sakata and Berg [23],

$$
D_{s} \approx 3 \times 10^{-8} \mathrm{~m}^{2} / \mathrm{s} .
$$

There are various options for our choice of velocity scale. For example, we could use the velocity $U_{0}$ from the formula for $L$, or we could use the average velocity during the opening phase, or we could pick one of the intrinsic velocity scales in the problem (determined by setting one of the dimensionless groups to unity). We choose to use the average velocity, $U_{a v}=0.044 \mathrm{~m} / \mathrm{s}$ which is appropriate for the blink, but will be higher than the intrinsic velocity induced by Marangoni effects after the upper eyelid has stopped moving. A consequence of this choice of velocity scale is that the capillary number is smaller than we would have anticipated. The dimensional parameter $h_{l}$ is the distance between the bottom surface of the upper eyelid and the eyeball surface. In keeping with papers such as Maki et al[13], we suppose that this thickness is of the order of $3 \mu \mathrm{m}$.

Using these parameters, we determine that

$$
\begin{array}{ccccc}
\epsilon=10^{-3}, & \mathrm{St}=1.7 \times 10^{-2}, & \mathrm{Re}=3.38 \times 10^{2}, & \mathrm{Ca}=1.27 \times 10^{-3}, & \\
\mathrm{Ma}=17.5, & \mathrm{Ev}=3.4 \times 10^{-4}, & \mathrm{Pe}=1.5 \times 10^{4}, & \mathcal{H}=100, & \mathcal{H}_{l}=0.3 .
\end{array}
$$

It is clear from the size of $\epsilon^{2}$ Re that inertia may be neglected, from the size of Pe that surface diffusion may be neglected, and from the size of Ev that evaporation can be neglected. Further, despite the evidence in Jones et al[11] that one should account for liquid flow under the eyelid, we choose to neglect the effect, based on the size of $\mathcal{H}_{l} / \mathcal{H}$; this giving us the worst possible case where no liquid is supplied to the tear film from under the upper eyelid. Since the focus of our investigation is how the presence of the polar lipid layer alters the behaviour of the tear film, we choose to retain both the capillary term $\epsilon^{3} / \mathrm{Ca}$ and the Marangoni term $\epsilon \mathrm{Ma}$, as well as the gravitational term. Retaining the capillary term might look dubious, given its size, but remember that, for small times, the film thickness is large, and also the horizontal distance is small, and we expect this term to have a significant influence on the film's evolution. We write $\mathcal{C}=\epsilon^{3} / \mathrm{Ca}$, $\mathcal{B}=\mathrm{St} / \mathcal{C}$ (the Bond number) and $\mathcal{M}=\epsilon \mathrm{Ma}$.

We substitute perturbation expansions for our dependent variables in terms of powers of $\epsilon$, and formulate the leading-order problem. The pressure is found to be constant across the film and, from (16b) the pressure gradient is found to be

$$
p_{x}=-\mathcal{C} h_{x x x} .
$$

Consequently from (15c) the liquid velocity is given by

$$
u=-\mathcal{C}\left(h_{x x x}-\mathcal{B}\right)\left(\frac{y^{2}}{2}-h y\right)-\mathcal{M} \Gamma_{x} y,
$$

and thus the average velocity, $\bar{u}$ is given by

$$
\bar{u}=\frac{1}{3} \mathcal{C} h^{2}\left(h_{x x x}-\mathcal{B}\right)-\frac{1}{2} \mathcal{M} \Gamma_{x} h,
$$


and the surface velocity at $y=h$ is

$$
u_{s}=\frac{1}{2} \mathcal{C} h^{2}\left(h_{x x x}-\mathcal{B}\right)-\mathcal{M} \Gamma_{x} h .
$$

Obtaining $v$ by integrating (15a) and applying the kinematic boundary condition (16a) gives,

$$
h_{t}+(\bar{u} h)_{x}=0 .
$$

While, neglecting both surface diffusion and evaporation for the reasons described earlier, (17) becomes

$$
\Gamma_{t}+\left(u_{s} \Gamma\right)_{x}=0 .
$$

The nondimensionalised boundary conditions read

$$
\begin{aligned}
& \bar{u}=0, \quad u_{s}=0, \quad h=\mathcal{H}, \quad \text { at } \quad x=0, \\
& \bar{u}=\dot{L}, \quad u_{s}=\dot{L}, \quad h=\mathcal{H}, \quad \text { at } \quad x=L,
\end{aligned}
$$

while the initial conditions are

$$
L(0)=\delta, \quad \Gamma(x, 0)=1, \quad h(x, 0)=\mathcal{H} .
$$

In summary, our nondimensionalised model comprises two coupled nonlinear partial differential equations for $h$ and $\Gamma$ on $0 \leq x \leq L(t)$,

$$
\begin{aligned}
h_{t}+\frac{1}{3} \mathcal{C}\left(h^{3}\left(h_{x x x}-\mathcal{B}\right)\right)_{x}-\frac{1}{2} \mathcal{M}\left(h^{2} \Gamma_{x}\right)_{x} & =0, \\
\Gamma_{t}+\frac{1}{2} \mathcal{C}\left(h^{2} \Gamma\left(h_{x x x}-\mathcal{B}\right)\right)_{x}-\mathcal{M}\left(h \Gamma \Gamma_{x}\right)_{x} & =0,
\end{aligned}
$$

with

$$
\begin{array}{cl}
h_{x x x}=\mathcal{B}, \quad \Gamma_{x}=0, \quad h=\mathcal{H}, \quad \text { on } \quad x=0, \\
h_{x x x}=\mathcal{B}+\frac{6 \dot{L}}{\mathcal{C H} \mathcal{H}^{2}}, \quad \Gamma_{x}=\frac{2 \dot{L}}{\mathcal{M H}}, \quad h=\mathcal{H}, \quad \text { on } \quad x=L(t),
\end{array}
$$

and

$$
L(0)=\delta, \quad \Gamma(x, 0)=1, \quad h(x, 0)=\mathcal{H},
$$

where we have used (27b) and (27c) to re-write (30a) and (30b) as boundary conditions for $h$ and $\Gamma$.

The coupled equations (32a), (32b) with boundary conditions (32cd) and initial conditions (32e) along with the equation for the eyelid position (19) specify the complete model for the dynamics of the thickness of the tear film and the concentration of lipid. In the sections that follow we will examine the behaviour of the system and its dependence on the capillary and Marangoni parameters.

In Section 5 we present results from a numerical solution of these equations, while in Section 6 we formulate an asymptotic solution to the problem for early time and then compare this with the numerics. We keep in mind that our ultimate goal is to examine whether the Marangoni traction exerted by non-uniform distribution of the lipid can cause the tear film to continue to move upwards after the upper eyelid has stopped moving. 



Figure 4: Numerical solution of (32) showing $h$ (top row) and $\Gamma$ (bottom row) for the dynamics of the early (left), intermediate (middle), and late (right) stages. The motion of the upper eyelid is shown in the early plots only. System parameters were $\mathcal{H}=100, \mathcal{C}=7.8 \times 10^{-7}, \mathcal{B}=21,800$, and $\mathcal{M}=1.8 \times 10^{-2}$.

\section{Behaviours observed from numerical simulations}

In order to obtain the parameter-dependence and general behaviours of system (32), we perform numerical simulations of the system. We compute the solution to this problem using a second-order accurate finite-difference scheme on a uniform spatial grid. For convenience we use a coordinate transformation to map the moving boundary problem (32) to a problem on a fixed computational domain (see appendix). Time-evolution is done in terms of an implicit backward Euler discretization and a Newton-Raphson method is used to solve the resulting nonlinear equations.

Figure 4 shows profiles of the tear film thickness and the lipid concentration at different times during the simulation. We observe that there are three distinct stages in the dynamics:

(a) Early stage: (left-hand column of Figure 4) As the eye begins opening, the film thins with a roughly parabolic profile whose minimum is steadily decreasing. The lipid evolves a minimum in concentration which has a position which roughly corresponds with the minimum in film thickness.

(b) Intermediate stage: (central column of Figure 4) The minimum film thickness has reached the capillary lengthscale and the fluid is collected in two quasi-steady menisci at the upper and lower eyelids. A thin film of liquid evolves between the two menisci, which is thinner near the upper eyelid than the lower one. The lipid becomes trapped in the two menisci, with a small amount on the surface of the thin film. 

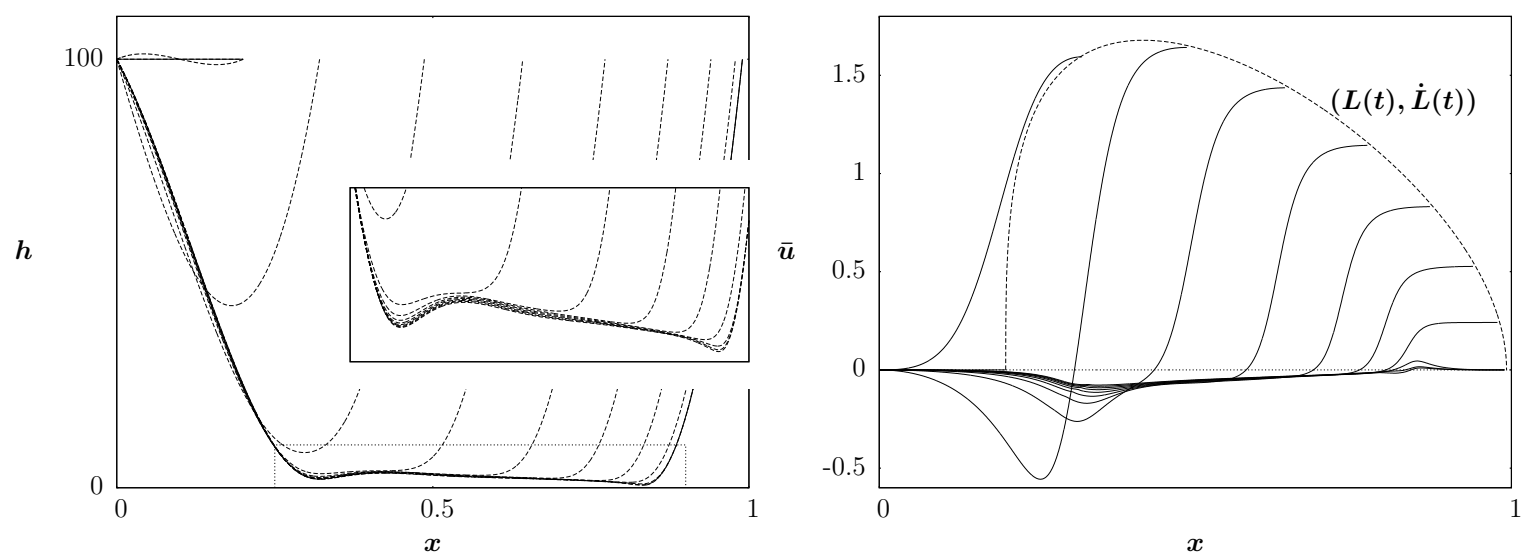

Figure 5: (Left) Comparison of tear film profiles produced by model (32a-e) with the influence of lipid (solid curves), and by model (33) (dashed) with no lipid (dashed). The inset shows details of the structure of the deposited thin film. (Right) Depth-averaged film velocity $\bar{u}$ (27b) produced by model (32a-e); the dashed envelope curve indicates the motion of the upper eyelid.

(c) Later stage: (right-hand column of Figure 4) After the upper eyelid has stopped moving the thin tear film between the menisci continues to slowly evolve, as does the lipid concentration. The concentration of lipid on the thin film is much lower than in the menisci, resulting in a much higher local surface tension. The lipid can be seen to be spreading back onto the thin film from both menisci, and, in this parameter regime, the concentration of lipid on the upper meniscus is smaller than that on the lower meniscus.

The behaviours in stages $(\mathrm{a}, \mathrm{b})$ have been computed in previous studies of tear films where the lipid component has been neglected, for example in [3]. Without lipid, (32) reduces to the simpler model,

$$
h_{t}+\frac{1}{3} \mathcal{C}\left(h^{3}\left(h_{x x x}-\mathcal{B}\right)\right)_{x}=0, \quad h(0)=h(L)=\mathcal{H}, \quad h_{x x x}(0)=\mathcal{B}, \quad h_{x x x}(L)=\mathcal{B}+\frac{3 \dot{L}}{\mathcal{C H}^{2}}
$$

Note that the boundary condition $h_{x x x}(L)$ is modified to satisfy the no-flux condition for this case. Figure 5(left) shows the the dynamics of (33) qualitatively matches the structure of the solution from the full system. However, careful examination of the thin film deposited on the eye (see inset) shows that the solution with the lipid is always thicker than the corresponding pure liquid film. This suggests that the presence of the lipid is beneficial in preventing extreme thinning of the film which may lead to dry-eye. Film rupture instabilities (with $h \rightarrow 0$ in finite time) observed in previous studies $[3,26,12,11]$ for $(33)$ have not been an issue in our study of $(32)$.

A physical analogy can be drawn between stage (b) and the Landau-Levich problem (see, eg [15]) for a solid plate being drawn out of a bath of viscous fluid $[12,26]$. The classic result for the drag-out problem is that the thickness of the film coating the plate scales with the capillary number to the two-thirds, or $h=O\left(\mathcal{C}^{-2 / 3}\right)$ in our notation. Figure 6 shows how the film thickness in the middle of the domain scales with the capillary number. System (32) was solved with a range of $\mathcal{C}$ values; the problem was also solved with $\mathcal{B}=0$, i.e. neglecting gravity, appropriate for a horizontal eye orientation. It is interesting to note that the presence of gravity causes additional thinning and drainage into the lower meniscus. These results are compared against computations from model 


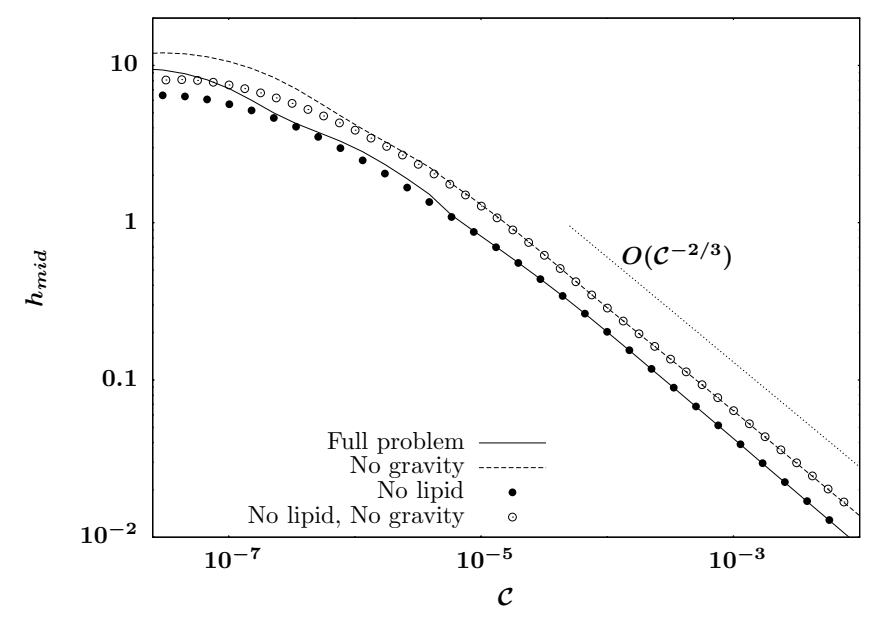

Figure 6: Plot of the film thickness in the middle of the domain at $t=2, h_{\text {mid }}$ for (32) and (33) with and without gravitational draining over a range of different values for the surface tension (inverse capillary number $\mathcal{C}$ ). The dotted line indicates the prediction of the behaviour of $h_{m i d}$ for the drag out problem, namely $\mathcal{C}^{-2 / 3}$.

(33), without lipid. For larger values of $\mathcal{C}$, the influence of the lipid appears negligible and the Landau-Levich scaling is obtained. For smaller values of $\mathcal{C}$ (the regime appropriate to tear films) the influence of lipids in increasing the film thickness becomes significant.

As noted above, when the surface of the eye is oriented vertically and the influence of gravity is present then the film will be thinned by an additional draining component to the flow. In the very early stages of the dynamics, this yields a local maximum of the film thickness and lipid concentration near the lower eyelid, see Figure 4(left), but this is a very short-time transient behaviour. The lasting influence of gravity is to create a stronger gradient of the thickness of the deposited thin film, favoring the meniscus at the lower eyelid over that at the upper eyelid, see Figures 4(right) and 5. A negative film velocity is required to do this, as seen in Figure 5(right).

The scaled Marangoni parameter $\mathcal{M}$ describes the coupling of the lipid concentration to the film thickness; the associated behaviours are less straightforward. Formally, setting $\mathcal{M}=0$ in (32a) yields the same PDE for $h(x, t)$ as (33). However if the eyelid is moving, then the boundary conditions for $h$ are different. For $\mathcal{M}>0$, distributions of lipid as shown in Figure 4 generate a gradient in the surface tension which promotes fluid flow from the menisci into the deposited thin film. Hence decreasing $\mathcal{M} \rightarrow 0$ can be expected to reduce the thickness of the film; indeed simulations show that a height profile similar to the one produced by (33) is obtained, as shown in Figure 7 (left). Yet, increasing $\mathcal{M}$ does not indefinitely increase the thickness of the central film; observe the approximate saturation in maximum height in Figure 7 (left). To understand this, we turn to (32b) where $\mathcal{M}$ has the role of a diffusion coefficient controlling the rate of spreading of $\Gamma$. If $\mathcal{M}$ is large then we expect $\Gamma$ to rapidly equilibrate to a uniform distribution and so we see that increasing $\mathcal{M}$ decreases the variation in lipid concentration. A consequence of this is to reduce the flux of fluid in equation (32a) into the central region. Figure 7 (right) show the nearly constant lipid profile at $t=2$ with large $\mathcal{M}$ and the formation of concentration plateaus at the menisci with sharpening transition layers for $\mathcal{M} \rightarrow 0$. It also demonstrates that it is possible to get more lipid on the upper meniscus than on the lower one, at least at this transient time. However, we note 

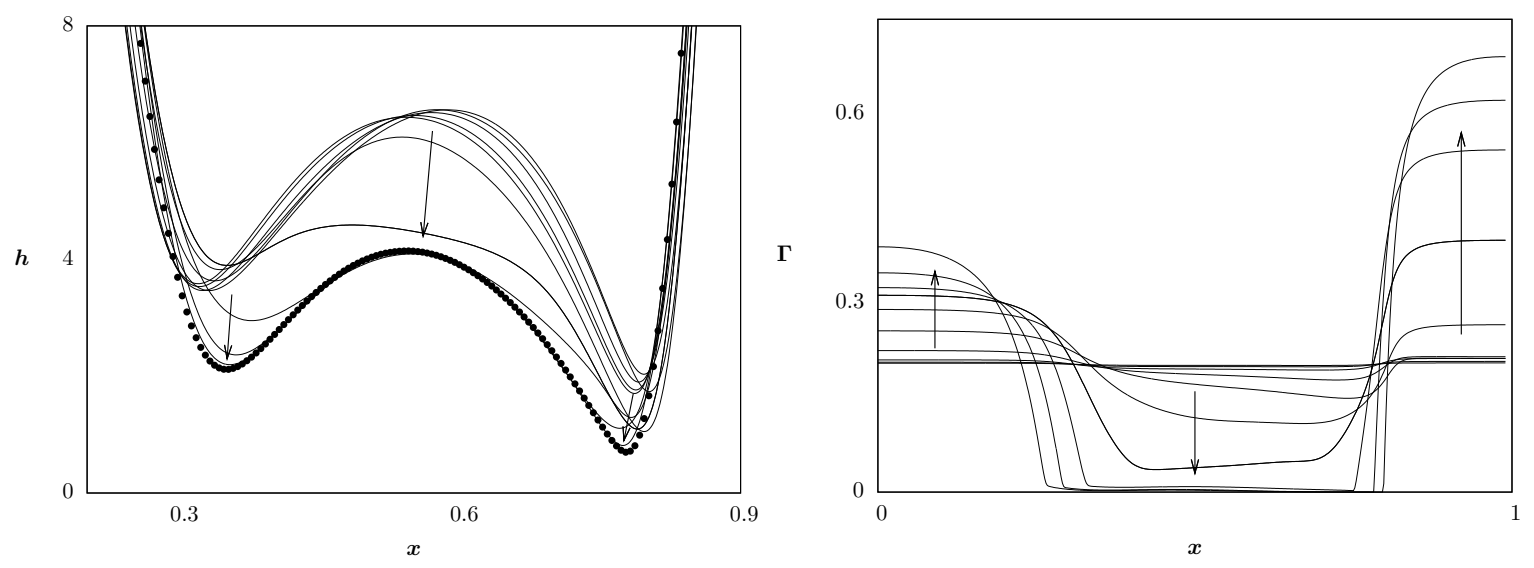

Figure 7: (left) Film height profiles at $t=2$ for various $\mathcal{M}$. Arrows indicate the trend for decreasing $\mathcal{M} \rightarrow 0$. Dotted curve is the height profile from (33). (right) Corresponding lipid concentration profiles.

that, while $\Gamma_{x} \rightarrow 0$ as $\mathcal{M}$ increases, the product $\mathcal{M} \Gamma_{x}$ is not everywhere small, with the result that the surface velocity does not vanish.

To describe transport in the late stage dynamics of the film, we reconsider the velocities $(27 \mathrm{~b})$ and $(27 \mathrm{c})$. We eliminate the competing draining due to gravity by setting $\mathcal{B}=0$ and plot the depth-averaged and surface velocities in Figure 8, for $t=1$ (which is after the eyelid has stopped moving). Our first observation is that the liquid is still moving despite the eyelids being fixed (and recall that we have neglected fluid inertia). This motion is driven by the interplay between capillary forces trying to drain the film into the menisci, and the Marangoni forces, trying to move liquid from the upper menisci into the film.

Figure 8(top left) shows the surface velocity. The parameter values used in Figure 4 give the bold curve. We see that there are regions where the fluid on the surface is moving up the eye, for example, from the lower eyelid out into the film, and regions where the fluid on the surface is moving down the eye, for example, from the upper meniscus into the film. Increasing the value of the Marangoni number results in the region of the fluid surface where the velocity is positive increasing in size, which tells us that the effect of the lipid is to drag the liquid up the eye. We note the interesting behaviour that increasing the Marangoni number also results in a change in the number of stagnation points on the surface, from three to one. Figure 8(top right) shows the corresponding values of the average velocity. Here, with our baseline parameter values we see, as expected, that the liquid is pulled out of the thin film and into both menisci (as a result of the negative pressure in the menisci generated from their curvature). As the Marangoni number is increased, the influence of the menisci decreases, and bulk fluid starts to be transported up the eye too. We note the interesting behaviour that, as the Marangoni number increases, a region just below the upper meniscus develops in which the fluid flows away from the meniscus. In Figure 8 (bottom left) and Figure 8 (bottom right), we see how the limit $\mathcal{M} \rightarrow 0$ compares with the " $\mathcal{M}=0$ " (no lipid) case. We see that the average velocity appears to match well, but that the surface velocities are different, as expected (recall that the boundary conditions on the surface velocity are different in each case).

As suggested by Figure 5(left), the presence of lipid increases the thickness of the tear film. 

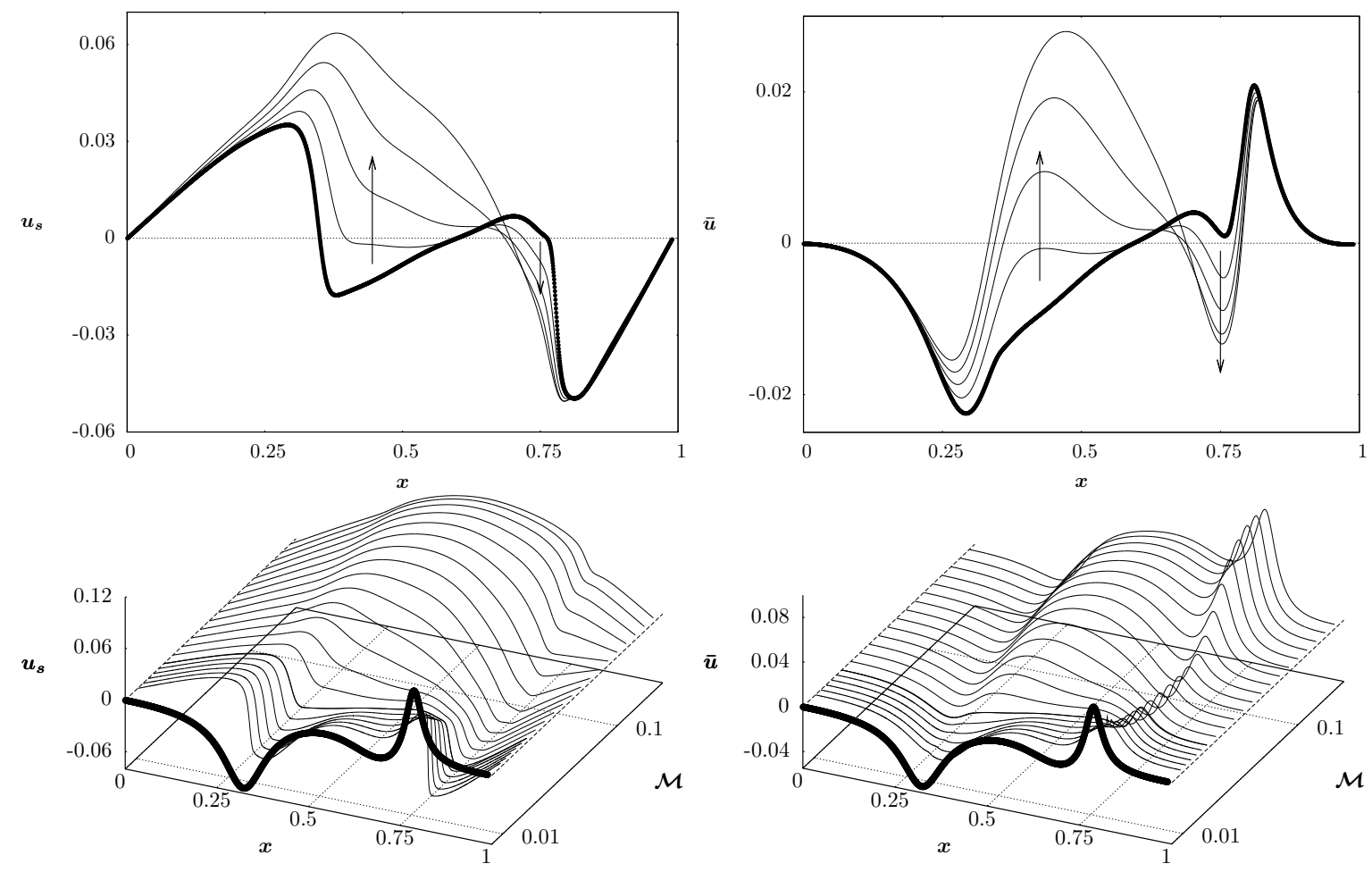

Figure 8: (Top row) The influence of increasing $\mathcal{M}$ on the surface velocity (left) and bulk velocity (right) evaluated at $t=1$ relative to the simulations from Fig. 4 (bold curves). (Bottom row) surface plots for surface and bulk velocities over a wider range of $\mathcal{M}$ compared to the no-lipid case (33) (bold curves). 


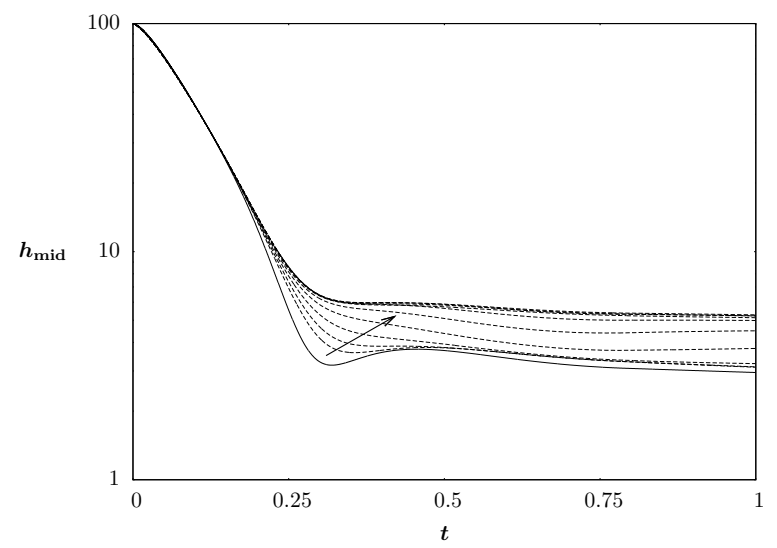

Figure 9: Variation in time of the thickness of the tear film in the middle of the eye with increasing Marangoni numbers.

In Figure 9, we investigate this further by showing the thickness of the midpoint of the tear film for various values of the Marangoni parameter. We see that the film thickness increases as $\mathcal{M}$ increases, suggesting that the lipid reduces the likelihood of film rupture.

In this section we have looked at the evolution of the tear film and shown that the presence of the lipid layer causes an upward motion of the free-surface of the liquid layer. During the initial stages of opening, the film thickness appears to be roughly parabolic. In the next section we examine the initial stages of the evolution asymptotically.

\section{Asymptotic behaviour of the solution}

At the start of the blink, the eyelids are close together and the film thickness is everywhere large. We examine this behaviour by setting

$$
h=\mathcal{H} \bar{h}, \quad x=\delta \bar{x}, \quad L=\delta \bar{L}, \quad \Gamma=\bar{\Gamma}, \quad t=\bar{t},
$$

with $\bar{L}(0)=1$, which results in the following set of equations

$$
\begin{aligned}
& \bar{h}_{\bar{t}}+\frac{1}{3} \mathbb{C}\left(\bar{h}^{3}\left(\bar{h}_{\bar{x} \bar{x} \bar{x}}-\mathbb{B}\right)\right)_{\bar{x}}-\frac{1}{2} \mathbb{M}\left(\bar{h}^{2} \bar{\Gamma}_{\bar{x}}\right)_{\bar{x}}=0, \\
& \bar{\Gamma}_{\bar{t}}+\frac{1}{2} \mathbb{C}\left(\bar{h}^{2} \bar{\Gamma}_{\bar{h}}\left(\bar{h}_{\bar{x} \bar{x} \bar{x}}-\mathbb{B}\right)\right)_{\bar{x}}-\mathbb{M}\left(\bar{h} \bar{\Gamma} \bar{\Gamma}_{\bar{x}}\right)_{\bar{x}}=0,
\end{aligned}
$$

with boundary conditions at $\bar{x}=0$ :

$$
\bar{h}_{\bar{x} \bar{x} \bar{x}}=\mathbb{B}, \quad \bar{\Gamma}_{\bar{x}}=0, \quad \bar{h}=1,
$$

and at $\bar{x}=\bar{L}(\bar{t})$ :

$$
\bar{h}_{\bar{x} \bar{x} \bar{x}}=\mathbb{B}+\frac{6}{\mathbb{C}} \frac{d \bar{L}}{d \bar{t}}, \quad \bar{\Gamma}_{\bar{x}}=\frac{2}{\mathbb{M}} \frac{d \bar{L}}{d \bar{t}}, \quad \bar{h}=1,
$$

and initial conditions at $\bar{t}=0$ :

$$
\bar{h}=1, \quad \bar{\Gamma}=1,
$$


where

$$
\mathbb{C}=\frac{\mathcal{C H}^{3}}{\delta^{4}}, \quad \mathbb{M}=\frac{\mathcal{M H}}{\delta^{2}}, \quad \mathbb{B}=\frac{\mathcal{B} \delta^{3}}{\mathcal{H}} .
$$

Using the same parameter values as before yields $\mathbb{C} \approx 490, \mathbb{M} \approx 44$, and $\mathbb{B} \approx 1.7$.

We take $\mathbb{B}=O(1)$ and consider two asymptotic regimes in the following subsections: (i) with $\mathbb{C}=O(\mathbb{M})$ and both being $\gg O(1)$, and (ii) with $\mathbb{C} \gg \mathbb{M}$ and $\mathbb{M}=O(1)$.

\section{1 $\mathbb{C}, \mathbb{M} \gg O(1)$}

We let $\chi=\mathbb{M} / \mathbb{C}$ be fixed and take the limit $\mathbb{C} \rightarrow \infty$. Our small parameter is $\mathbb{C}^{-1}$ and we seek expansions of the dependent variables of the form $\bar{h}=\bar{h}_{0}+\mathbb{C}^{-1} h_{1}+O\left(\mathbb{C}^{-2}\right), \bar{\Gamma}=\Gamma_{0}+\mathbb{C}^{-1} \bar{\Gamma}_{1}+$ $O\left(\mathbb{C}^{-2}\right)$. The leading-order problem becomes:

$$
\begin{gathered}
\left(\frac{1}{3} \bar{h}_{0}^{3}\left(\bar{h}_{0 \bar{x} \bar{x} \bar{x}}-\mathbb{B}\right)-\frac{1}{2} \chi \bar{h}_{0}^{2} \bar{\Gamma}_{0 \bar{x}}\right)_{\bar{x}}=0, \\
\left(\frac{1}{2} \bar{h}_{0}^{2} \bar{\Gamma}_{0}\left(\bar{h}_{0 \bar{x} \bar{x} \bar{x}}-\mathbb{B}\right)-\chi \bar{h}_{0} \bar{\Gamma}_{0} \bar{\Gamma}_{0 \bar{x}}\right)_{\bar{x}}=0,
\end{gathered}
$$

with boundary conditions

$$
\begin{array}{lllll}
\bar{h}_{0}=1, & \bar{h}_{0 \bar{x} \bar{x} \bar{x}}=\mathbb{B}, & \bar{\Gamma}_{0 \bar{x}}=0, & \text { at } & \bar{x}=0, \\
\bar{h}_{0}=1, & \bar{h}_{0 \bar{x} \bar{x} \bar{x}}=\mathbb{B}, & \bar{\Gamma}_{0 \bar{x}}=0, & \text { at } & \bar{x}=\bar{L} .
\end{array}
$$

We integrate (37a) and (37b), apply the boundary conditions and rearrange to find that

$$
\bar{h}_{0 \bar{x} \bar{x} \bar{x}}=\mathbb{B}, \quad \bar{\Gamma}_{0 \bar{x}}=0 .
$$

Thus $h_{0}$ has the solution

$$
\bar{h}_{0}(\bar{x}, \bar{t})=1-6 \eta(1-\eta)\left(1-\frac{1}{\bar{L}}\right)+\frac{\bar{L}^{3} \mathbb{B}}{12} \eta(1-\eta)(1-2 \eta), \quad \eta=\frac{\bar{x}}{\bar{L}(\bar{t})},
$$

where we have appealed to global conservation of mass in order to determine one of the constants of integration. Equation (38) also dictates that $\bar{\Gamma}_{0}$ is a function of time only. In order to determine $\bar{\Gamma}_{0}$ we use the global conservation of lipid, yielding

$$
\bar{\Gamma}_{0}(\bar{t})=\frac{1}{\bar{L}(\bar{t})} .
$$

This result effectively gives us $\bar{\Gamma}_{0}(\bar{t})$ as the mean lipid concentration over the domain. To obtain information about the spatial structure of the lipid concentration we proceed to the next order in the expansion:

$$
\begin{gathered}
\bar{h}_{0 \bar{t}}+\left(\frac{1}{3} \bar{h}_{0}^{3} \bar{h}_{1 \bar{x} \bar{x} \bar{x}}-\frac{1}{2} \chi \bar{h}_{0}^{2} \bar{\Gamma}_{1 \bar{x}}\right)_{\bar{x}}=0, \\
\bar{\Gamma}_{0 \bar{t}}+\left(\frac{1}{2} \bar{h}_{0}^{2} \bar{\Gamma}_{0} \bar{h}_{1 \bar{x} \bar{x} \bar{x}}-\chi \bar{h}_{0} \bar{\Gamma}_{0} \bar{\Gamma}_{1 \bar{x}}\right)_{\bar{x}}=0,
\end{gathered}
$$

with boundary conditions

$$
\begin{array}{ccc}
\bar{h}_{1}=0, \quad \bar{h}_{1 \bar{x} \bar{x} \bar{x}}=0, & \bar{\Gamma}_{1 \bar{x}}=0, \quad \text { at } \quad \bar{x}=0, \\
\bar{h}_{1}=0, \quad \bar{h}_{1 \bar{x} \bar{x} \bar{x}}=6 \frac{d \bar{L}}{d \bar{t}}, & \bar{\Gamma}_{1 \bar{x}}=\frac{2}{\chi} \frac{d \bar{L}}{d \bar{t}}, \quad \text { at } \quad \bar{x}=\bar{L} .
\end{array}
$$



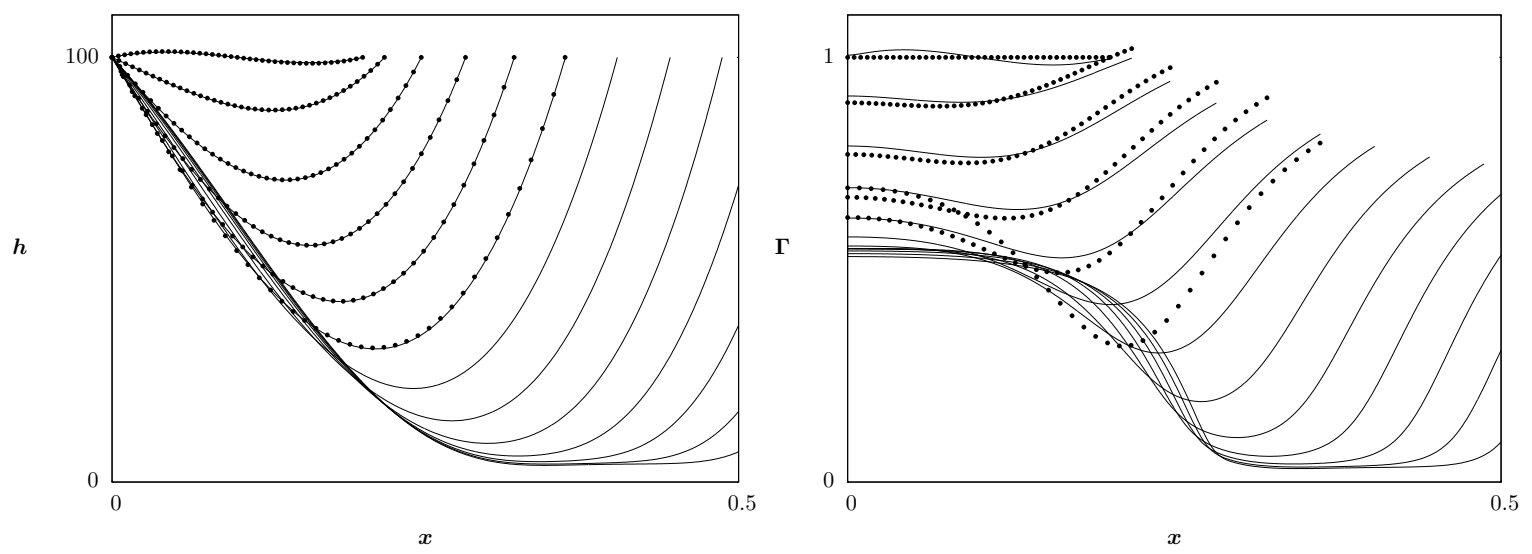

Figure 10: Comparison of the asymptotic model from section 6.1 (dotted) with the numerical solution from Fig. 4 (solid curves): (left) excellent agreement of the height profiles with (39), (right) reasonable qualitative correspondence for the lipid concentration.

We integrate these equations and apply the boundary conditions at $\bar{x}=0$ to find that

$$
\begin{gathered}
\int_{0}^{\bar{x}} \bar{h}_{0 \bar{t}} d \bar{x}^{\prime}+\frac{1}{3} \bar{h}_{0}^{3} \bar{h}_{1 \bar{x} \bar{x} \bar{x}}-\frac{1}{2} \chi \bar{h}_{0}^{2} \bar{\Gamma}_{1 \bar{x}}=0, \\
\bar{x} \bar{\Gamma}_{0 \bar{t}}+\frac{1}{2} \bar{h}_{0}^{2} \bar{\Gamma}_{0} \bar{h}_{1 \bar{x} \bar{x} \bar{x}}-\chi \bar{h}_{0} \bar{\Gamma}_{0} \bar{\Gamma}_{1 \bar{x}}=0 .
\end{gathered}
$$

We then rearrange to get differential equations for $\bar{h}_{1}$ and $\bar{\Gamma}_{1}$, which read

$$
\begin{gathered}
\bar{h}_{1 \bar{x} \bar{x} \bar{x}}=-\frac{6 \bar{x}}{\bar{h}_{0}^{2} \bar{L}} \frac{d \bar{L}}{d \bar{t}}-\frac{12}{\bar{h}_{0}^{3}} \int_{0}^{\bar{x}} \bar{h}_{0 \bar{t}} d \bar{x}^{\prime}, \\
\bar{\Gamma}_{1 \bar{x}}=-\frac{4 \bar{x}}{\chi \bar{h}_{0} \bar{L}} \frac{d \bar{L}}{d \bar{t}}-\frac{6}{\chi \bar{h}_{0}^{2}} \int_{0}^{\bar{x}} \bar{h}_{0 \bar{t}} d \bar{x}^{\prime},
\end{gathered}
$$

These are two decoupled boundary value problems (ODEs in $\bar{x}$ ) for $\bar{h}_{1}$ and $\bar{\Gamma}_{1}$ on $0 \leq \bar{x} \leq \bar{L}$; they depend only parametrically on $\bar{t}$. Integrating them numerically is straightforward and the solutions can be obtained via shooting to satisfy the conditions

$$
\bar{h}_{1}(0)=\bar{h}_{1}(\bar{L})=0, \quad \int_{0}^{\bar{L}} \bar{h}_{1} d \bar{x}=0, \quad \int_{0}^{\bar{L}} \bar{\Gamma}_{1} d \bar{x}=0 .
$$

Note that all terms on the right hand sides of (43) are proportional to $d \bar{L} / d \bar{t}$, so these corrections are strictly due to the motion of the eyelid (when motion stops, these become zero). In Figure 10 we compare solutions to the asymptotic problem with those calculated numerically for the full problem. We can see that the agreement between the two is excellent for the film thickness, but that the agreement is less good for the lipid concentrations, which deviate even for short times.

\section{$6.2 \mathbb{C} \gg \mathbb{M}$}

Motivated by the need to get a more accurate description of the lipid concentration we consider a different limiting approximation, where we assume that $\mathbb{C} \gg \mathbb{M}$. Again, taking $\mathbb{C} \rightarrow \infty$ and 

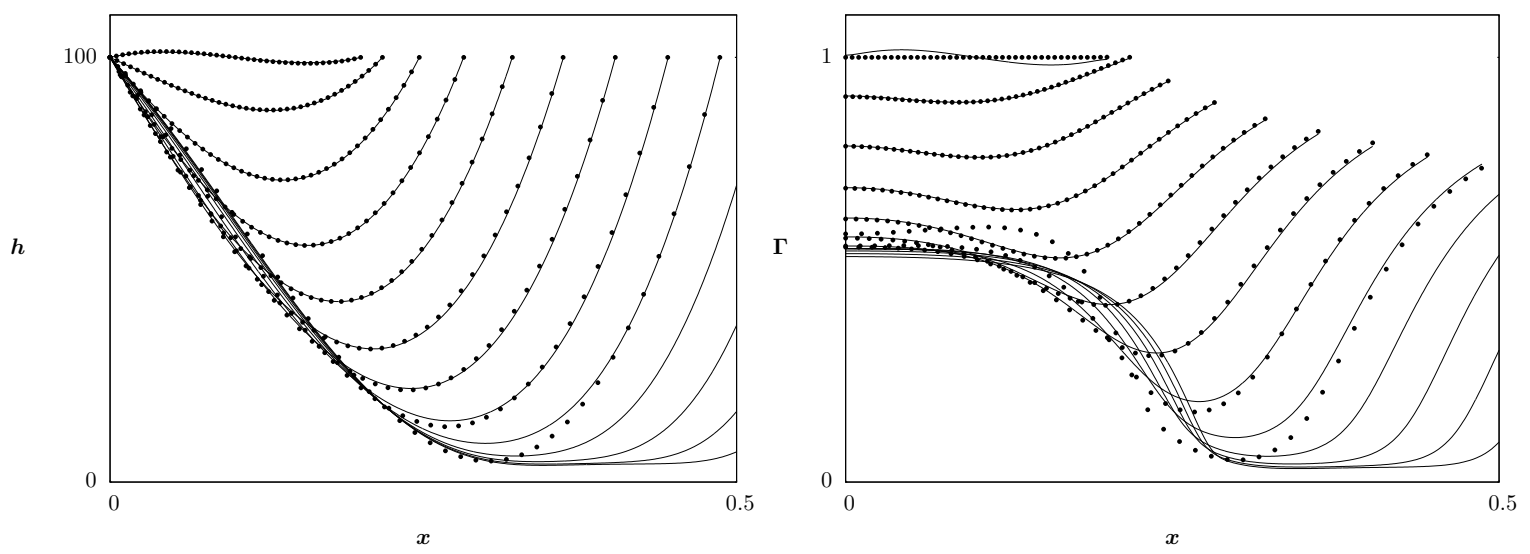

Figure 11: Comparison of the asymptotic model from section 6.2 (dotted) with the numerical solution from Fig. 4 (solid curves): far better agreement is observed than in Fig. 10.

expanding our dependent variables in powers of $\mathbb{C}^{-1}$, i.e. $\bar{h}=\bar{h}_{0}+\mathbb{C}^{-1} \bar{h}_{1}+O\left(\mathbb{C}^{-2}\right), \bar{\Gamma}=\bar{\Gamma}_{0}+$ $O\left(\mathbb{C}^{-1}\right)$. We find, at leading order, that

$$
\bar{h}_{0 \bar{x} \bar{x} \bar{x}}=\mathbb{B},
$$

which has the solution given by (39).

The next-order corrections to the two field equations and boundary conditions read

$$
\begin{gathered}
\bar{h}_{0 \bar{t}}+\frac{1}{3}\left(\bar{h}_{0}^{3} \bar{h}_{1 \bar{x} \bar{x} \bar{x}}\right)_{\bar{x}}-\frac{1}{2} \mathbb{M}\left(\bar{h}_{0}^{2} \bar{\Gamma}_{0 \bar{x}}\right)_{\bar{x}}=0, \\
\bar{\Gamma}_{0 \bar{t}}+\frac{1}{2}\left(\bar{h}_{0}^{2} \bar{\Gamma}_{0} \bar{h}_{1 \bar{x} \bar{x} \bar{x}}\right)_{\bar{x}}-\mathbb{M}\left(\bar{h}_{0} \bar{\Gamma}_{0} \bar{\Gamma}_{0 \bar{x}}\right)_{\bar{x}}=0, \\
\bar{h}_{1 \bar{x} \bar{x} \bar{x}}=0, \quad \bar{\Gamma}_{0 \bar{x}}=0, \quad \bar{h}_{1}=0 \quad \text { at } \quad \bar{x}=0, \\
\bar{h}_{1 \bar{x} \bar{x} \bar{x}}=6 \frac{d \bar{L}}{d \bar{t}}, \quad \bar{\Gamma}_{0 \bar{x}}=\frac{2}{\mathbb{M}} \frac{d \bar{L}}{d \bar{t}}, \quad \bar{h}_{1}=0 \quad \text { at } \quad \bar{x}=\bar{L},
\end{gathered}
$$

Integrating (46a) and applying the boundary conditions, we find that

$$
\frac{1}{3} \bar{h}_{0}^{3} \bar{h}_{1 \bar{x} \bar{x} \bar{x}}=\frac{1}{2} \mathbb{M} \bar{h}_{0}^{2} \bar{\Gamma}_{0 \bar{x}}-\int_{0}^{\bar{x}} \bar{h}_{0 \bar{t}} d \bar{x}^{\prime} .
$$

Subsequently by using (47) for $\bar{h}_{1 \bar{x} \bar{x} \bar{x}}$, equation (46b) can be written as and (46b) reduces to

$$
\bar{\Gamma}_{0 \bar{t}}-\frac{1}{4} \mathbb{M}\left(\bar{h}_{0} \bar{\Gamma}_{0} \bar{\Gamma}_{0 \bar{x}}\right)_{\bar{x}}-\frac{3}{2}\left(\frac{\bar{\Gamma}_{0}}{\bar{h}_{0}} \int_{0}^{\bar{x}} \bar{h}_{0 \bar{t}} d \bar{x}^{\prime}\right)_{\bar{x}}=0 .
$$

With $\bar{h}_{0}(x, t)$ given by (39), equation (48) is a nonlinear convection-diffusion equation for $\bar{\Gamma}_{0}$. If needed, $\bar{h}_{1}$ can then be found by solving (47). In Figure 11 we compare our new asymptotic approximation for $\bar{\Gamma}_{0}$ with the same numerical solution as in Figure 10. We see that there is much improved agreement between the asymptotic solution and the solution to the full problem and the agreement is sustained for longer. 


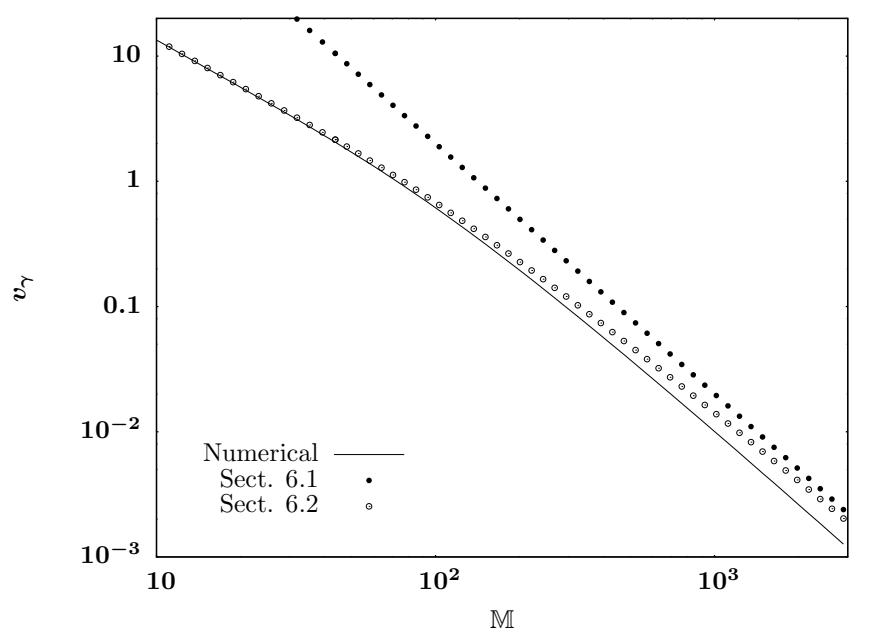

Figure 12: The dependence of a measure of spatial variation in the lipid distribution $v_{\Gamma}$ as a function of $\mathbb{M}$ at time $t=0.15$. The solid curve is from the numerical solution from Fig. 4, solid circles give the asymptotic model from Sect. 6.1 and open circles give the model from Sect. 6.2.

In order to evaluate how the spatial variation of the lipid profile changes with Marangoni parameter, we calculate

$$
v_{\Gamma}=\int_{0}^{L(t)} \Gamma_{x}^{2} d x .
$$

Examining the value of this integral at a fixed time $(t=0.15)$ over a range of values for $\mathbb{M}$ allows us to concisely compare the two asymptotic models with the full numerical simuation. The resulting curves are shown in Figure 12. full problem, the closed circles correspond to the solution presented in Section 6.1 while the open circles correspond to the solution presented in Section 6.2. We can easily see from this curve that the spatial variation in the lipid profile decreases as the Marangoni parameter increases, and that the second of these asymptotic solutions accurately captures the behaviour of the numerical solution to the full PDE system. The figure shows convergence of the asymptotic models in the limits of $\mathbb{M} \rightarrow \infty$ and $\mathbb{M} \rightarrow 0$ respectively as expected from Sects. 6.1 and 6.2 .

The asymptotic solution breaks down when the minimum in $h$ becomes zero (which, when $\mathbb{B}=0$, occurs when $\bar{L}=3$ and $\eta=1 / 2$, see appendix). In order to advance our asymptotic solution to later times, we must decompose the region into a thin film, a capillary static region, and a transition region between the two. This asymptotic decomposition will form the basis of future work.

\section{$7 \quad$ Discussion and conclusion}

In this paper we presented a mathematical model to describe the influence of lipid on the evolution of a tear film. We assumed that the lipid could be described as an insoluble surfactant which locally modified the surface tension. In the lubrication limit, our model reduced to a pair of coupled partial differential equations for the evolution of the thickness of the tear film and the concentration of the lipid. The key parameters in the model are the capillary number, the Bond number and the 
Marangoni number. We focus on the influence of the lipid on the evolution of the tear film by varying the Marangoni number.

We were able to draw an analogy between the drainage of our tear film and the classical "drag out" problem. In particular, we saw that the the film thickness follows the standard "capillary number to the two-thirds" rule established for other thin film drainage problems. However, we note that the operating regime for tear film drainage is away from this asymptotic limit of the model [12].

In the absence of lipid we see that the tear film evolves in the same manner as described by other authors, and that after the eyelid has stopped moving there is a small drainage flow which moves liquid from the thin films into the menisci. However, in the absence of flow from under the eyelids, our tear film does not "dry out". With lipid present, the motion of the upper eyelid generates a nonuniform concentration of lipid which generates a Marangoni stress on the air-liquid interface and modifies the underlying flow. We find that surface velocity increases as the Marangoni number increases, and thus that the presence of the lipid causes a flow that resists the drainage caused by the curvature of the meniscus. The resulting tear films are thicker as a consequence. We highlighted the interesting behaviour that increasing the Marangoni number alters the number of stagnation points on the air-liquid interface. We will further investigate this phenomenon in future work.

We examined the initial motion of the tear film using asymptotic methods, and solved the resulting system of equations to find an approximation for the film shape and the lipid concentration. In one asymptotic limit, these matched up well with the numerical solution of the full problem. The agreement between the two solutions worsened as the minimum thickness of the film became small: continuing this asymptotic approach will be future work.

In our model, we have see that reducing the Marangoni number decreases the tear film thickness and reduces the surface velocity. We recall that the Marangoni number is directly proportional to the initial concentration of lipid. Experimentalists concur that in dry eye sufferers the tear film thickness is smaller and the spreading of the lipid layer is slower than compared with those with normal eyes. We conclude that a reduction in the initial concentration of lipid generates flows which are consistent with experimental observations of dry eye, and thus we speculate that dry eye sufferers may have reduced (polar) lipid production.

However, in order to examine dry eye comprehensively, we need to return to the issue of evaporation, which crucially depends on the thickness of the nonpolar lipid layer (which provides a barrier to evaporation). Nonpolar lipids are present in much larger quantities than the polar component and reside as a contiguous layer above the aqueous interface. While the layer is small when compared with the aqueous layer, the lines seen in Figure 1 are the edge of the nonpolar lipid layer as it spreads (and experiments that look at lipid distribution only look at nonpolar lipids). Preliminary work modelling the polar lipid suggests that it cannot support shear and acts as an extensional layer. The resulting model comprises of four coupled nonlinear partial differential equations describing the thickness of the aqueous layer, the thickness of the nonpolar lipid layer, the velocity in the nonpolar lipid layer and the concentration of polar lipid. It is unclear from experiments whether the nonpolar lipid layer has a sharp "front", or whether the layer extends all the way across the eye surface, but in places is thinner than the experimentalists can resolve [1]. The boundary condition to be imposed at the advancing front of nonpolar lipid layer is crucial to determining how and where the evaporation takes place following a blink, provides nontrivial mathematical challenges, and is the subject of ongoing work. 
Our ultimate goal is to produce a mathematical model for a blink which incorporates both types of lipid, evaporation and the supply of water from under the lids, so that both aqueous-deficient and evaporative dry eye can be examined.

\section{Acknowledgments}

The authors are particularly grateful for extensive dialogue with Prof. Colin Please, useful comments by Dr Jim Oliver, and for the support and insight provided by their experimental collaborators, Prof. Anthony Bron and Dr John Tiffany. This work was partly funded by an EPSRC-funded MSc studentship, and is also partially based on work supported by award number KUK-C1-013-04 made by King Abdullah University of Science and Technology and NSF DMS CAREER grant 0239125 .

\section{A Asymptotics of the basic moving boundary problem}

Insights on numerical and analytical issues for the full tear film problem (32) can be gained by considering the basic moving boundary problem for the thin film equation. Consider (33) in the absence of the influences of the lipid and gravity, i.e. with $\mathcal{B}=0$,

$$
h_{t}+\frac{1}{3} \mathcal{C}\left(h^{3} h_{x x x}\right)_{x}=0, \quad h(0, t)=h(L(t), t)=\mathcal{H}, \quad h_{x x x}(0, t)=0, \quad h_{x x x}(L(t), t)=\frac{3 \dot{L}}{\mathcal{C H}^{2}}
$$

with initial condition $h(x, 0)=\mathcal{H}$ on $0 \leq x \leq L(0)$ at $t=0$. As done in other studies [11, 12, $7,13,3,4]$, for computations it is convenient to map the problem onto a fixed computational domain using the change of variables $x=L(t) \eta$, with $0 \leq \eta \leq 1$ and the normalized film thickness $h(x, t)=\mathcal{H} H(\eta, t)$, yielding the governing equation

$$
H_{t}-\frac{\dot{L}}{L} \eta H_{\eta}+\frac{\mathcal{H}^{3} \mathcal{C}}{3 L^{4}}\left(H^{3} H_{\eta \eta \eta}\right)_{\eta}=0
$$

with boundary conditions

$$
H(0, t)=H(1, t)=1, \quad H_{\eta \eta \eta}(0, t)=0, \quad H_{\eta \eta \eta}(1, t)=\frac{3 L^{3} \dot{L}}{\mathcal{H}^{3} \mathcal{C}},
$$

and initial condition $H(\eta, 0)=1$ on $0 \leq \eta \leq 1$. The statement of conservation of mass then takes the form

$$
\int_{0}^{1} H(\eta, t) d \eta=\frac{L(0)}{L(t)}
$$

It is helpful to multiply (51a) by $L(t)$ to produce an equation in conservation-law form $[10,9]$

$$
(L H)_{t}+\left(H\left[\frac{\mathcal{H}^{3} \mathcal{C}}{3 L^{3}} H^{2} H_{\eta \eta \eta}-\eta \dot{L}\right]\right)_{\eta}=0
$$

this form allows for numerical methods that are much more accurate in preserving the tear film mass than for (51a). Factoring $\dot{L}$ out of the flux, we can write the problem as

$$
(L H)_{t}+\dot{L}\left(H\left[\frac{1}{\alpha} H^{2} H_{\eta \eta \eta}-\eta\right]\right)_{\eta}=0,
$$




$$
H(0, t)=H(1, t)=1 \quad H_{\eta \eta \eta}(0, t)=0 \quad H_{\eta \eta \eta}(1, t)=\alpha,
$$

where

$$
\alpha \equiv \frac{3 L^{3} \dot{L}}{\mathcal{H}^{3} \mathcal{C}} .
$$

The parameter $\alpha$ is like a Peclet number (a ratio of convective to diffusive transport speeds) allowing us to compare the influences of the flow generated by the moving boundary vs. capillarity. Its value is time-dependent, but during the opening of the eye, it will be positive and bounded proportional to the maximum speed of the eyelid.

\section{A.1 Case 1: Behaviour for $\alpha \rightarrow 0$}

Consider the limit of small $\alpha$; this is the case where surface tension is the dominant effect and works to smooth out variations in the curvature. It is convenient to re-write (54a) as

$$
\left(H^{3} H_{\eta \eta \eta}\right)_{\eta}+\alpha\left[(L H)_{t}-\dot{L}(\eta H)_{\eta}\right]=0
$$

and expand the solution as a regular perturbation series, $H \sim H_{0}+\alpha H_{1}+O\left(\alpha^{2}\right)$. At leading order we get a quasi-static problem for $H_{0}$,

$$
H_{0 \eta \eta \eta}=0, \quad H_{0}(0)=H_{0}(1)=1, \quad H_{0 \eta \eta \eta}(0)=H_{0 \eta \eta \eta}(1)=0
$$

with the solution

$$
H_{0}(\eta, t)=1-6[1-\gamma]\left(\eta-\eta^{2}\right),
$$

where $\gamma(t)=L(0) / L(t) \leq 1$, see Fig. 13. Equation (39), that played the key role of leading order solution in both of the asymptotic models in section 6 (where $\alpha=O\left(10^{-3}\right)$ ), is a generalization of this solution. Requiring the film thickness predicted by (58) to remain positive gives an estimate of the duration of the "early stage" (described at the beginning of section 5 ) as times $0 \leq t \leq t_{0}$ such that $L(t)<3 L(0)$. Figure 13 shows very good agreement with numerical computed profiles during this regime. Profiles past the end of the early stage still show some resemblance to the positive truncation of the parabolic profile (58); see the last dotted profile in Figure 13.

\section{A.2 Case 2: Short-time behaviour for large $\alpha$}

For $\alpha \rightarrow \infty$ the film behaviour is primarily controlled by the motion of the moving eyelid. This limit is a singular perturbation as the highest-order spatial derivative term in (54a) has a vanishing coefficient. Moreover, from (54b) we expect formation of a boundary layer at the moving boundary, as the gradients of the film thickness vary rapidly there. Away from a neighborhood of $\eta=1$, the singular term can be neglected to leading order, yielding the problem

$$
\left(L H_{0}\right)_{t}-\dot{L}\left(\eta H_{0}\right)_{\eta}=0 \text {. }
$$

Solving via the method of characteristics yields $H_{0}(\eta, t)=\tilde{H}(L(t) \eta)$, i.e. a steady solution for $h(x)$. In fact by the initial conditions, $H_{0} \equiv 1$, uniform constant for $0 \leq \eta<1$. This state is maintained until the influence of the moving eyelid propagates into the film from $\eta=1$. We will not pursue the full matched asymptotic analysis of this behaviour; instead we will describe how the short-time dynamics can be approximated by a self-similar solution. 


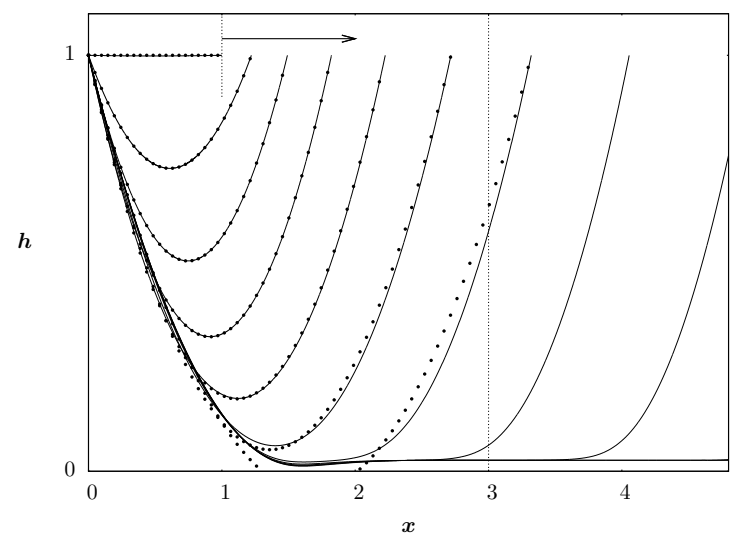

Figure 13: Numerically calculated solution profiles for (54ab) with $\mathcal{H}^{3} \mathcal{C}=1000$ and $L(t)=e^{t}$ at times $t=0,0.2,0.4, \cdots$ (solid curves) and predicted profiles given by (58) (dotted). The vertical line at $x=3$ indicates the end of the predicted "early stage" regime.

In this limit for short times, $t \rightarrow 0$, consider the general form for the boundary motion, $L(t) \sim$ $L_{0}+L_{1} t^{\beta}$ with $\beta \geq 1$. We write the solution of (54a) as

$$
H(\eta, t)=1+\phi(\eta, t), \quad \phi \ll 1,
$$

where from the initial conditions, $\phi(\eta, 0)=0$. For $t \rightarrow 0$, using $L \sim L_{0}, \dot{L} \sim \beta L_{1} t^{\beta-1}$, the linearized problem for $\phi$ is

$$
\phi_{t}-\frac{\beta L_{1} t^{\beta-1}}{L_{0}} \eta \phi_{\eta}+\frac{1}{\tilde{\alpha}} \phi_{\eta \eta \eta \eta}=0, \quad \phi(1)=0, \quad \phi_{\eta \eta \eta}(1)=\tilde{\alpha} \frac{\beta L_{1}}{L_{0}} t^{\beta-1},
$$

where $\tilde{\alpha}=3 L_{0}^{4} /\left(\mathcal{H}^{3} \mathcal{C}\right)$. Neglecting higher order terms as $t \rightarrow 0$, this problem has an asymptotic similarity solution of the form

$$
\phi(\eta, t)=t^{\beta-1 / 4} g(\zeta), \quad \zeta=(\eta-1),(\tilde{\alpha} / t)^{1 / 4}
$$

where $g(\zeta)$ satisfies

$$
\begin{gathered}
\left(\beta-\frac{1}{4}\right) g-\frac{\zeta}{4} \frac{d g}{d \zeta}+\frac{d^{4} g}{d \zeta^{4}}=0 \\
g(\zeta \rightarrow-\infty) \rightarrow 0 \quad g(0)=0 \quad g^{\prime \prime \prime}(0)=\tilde{\alpha}^{1 / 4} \frac{\beta L_{1}}{L_{0}} .
\end{gathered}
$$

The similarity function $g(\zeta)$ can be obtained numerical via a two-parameter shooting search. Calculation of the similarity solution can be obtained via a 2-parameter shooting search. Figure 14 shows good quantitative agreement with the similarity solution during the beginning of the motion of the upper eyelid.

\section{References}

[1] Bron A.J. and Tiffany J. M. Personal communication. 2008. 

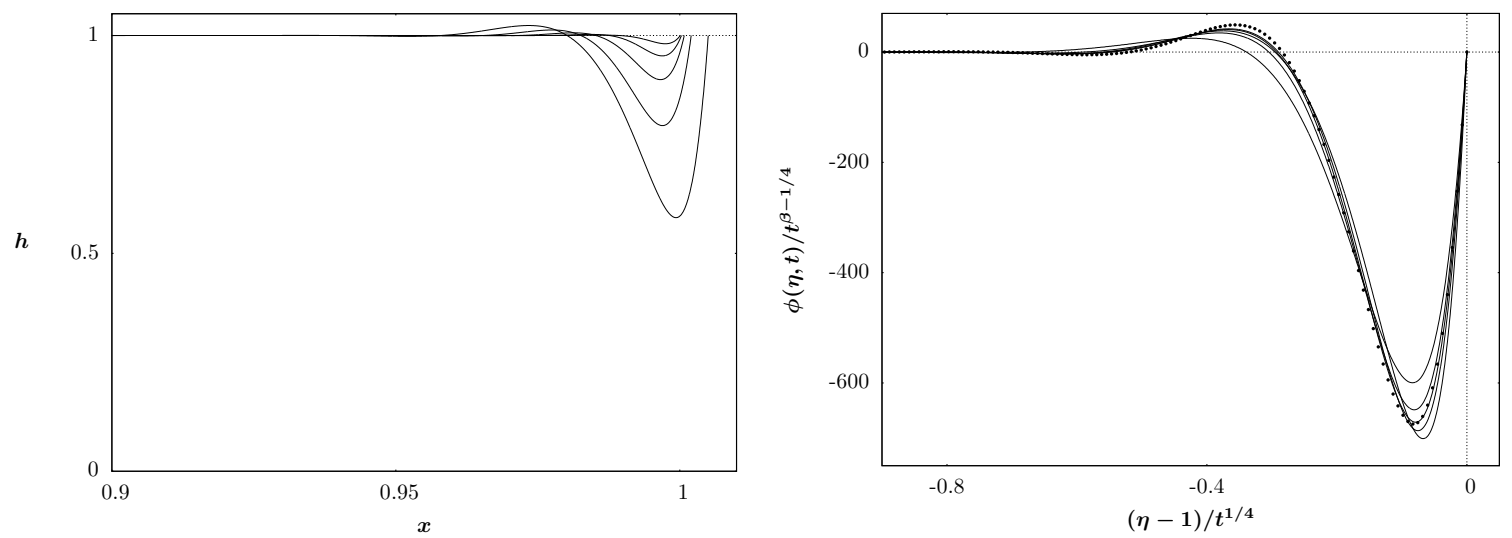

Figure 14: (Left) Numerically calculated solution profiles for (54ab) with $\mathcal{H}^{3} \mathcal{C}=10^{-4}$ and $L(t)=$ $1+100 t$ in a small neighborhood of the upper eyelid for short times and (right) the numerical profiles (solid curves) compared with $g(\zeta)$ (dotted) plotted in similarity variables.

[2] R. E. Berger and S. Corrsin. A surface tension gradient mechanism for driving the pre-corneal tear film after a blink. J. Biomech., 7(3):225-238, 1974.

[3] R. J. Braun and A. D. Fitt. Modelling drainage of the precorneal tear film after a blink. Math. Med. Biol., 20(1):1-28, 2003.

[4] R. J. Braun and P. E. King-Smith. Model problems for the tear film in a blink cycle: singleequation models. Journal of Fluid Mechanics, 586:465-490, 2007.

[5] C. J. W. Breward, , R. C. Darton, P. D. Howell, and J. R. Ockendon. The effect of surfactants on expanding free surfaces. Chem. Eng. Sci., 56:2867-2878, 2001.

[6] R. S. Burdon. Surface Tension and the Spreading of Liquids. Cambridge University Press, London, 1949.

[7] A. Heryudono, R. J. Braun, T. A. Driscoll, K. L. Maki, and L. P. Cooke. Single-equation models for the tear film in a blink cycle: Realistic lid motion. To be published in MBB Journal, 2007.

[8] F. J. Holly. Formation and rupture of the tear film. Exp. Eye Res., 15(5):515-525, 1973.

[9] T. C. Illingworth and I. O Golosnov. Numerical solutions of diffusion-controlled moving boundary problems which conserve solute. Journal of Computational Physics, 209:207-225, 2005.

[10] T. C. Illingworth, I. O. Golosnov, V. Gergely, and T. W. Clyne. Numerical modeling of transient liquid phase bonding and other diffusion controlled phase changes. Journal of Materials Science, 40:2505-2511, 2005.

[11] M. B Jones, D. L. S McElwain, G. R. Fulford, M. J. Collins, and A. P. Roberts. The effect of the lipid layer on tear film behaviour. Bull. Math. Biol., 68(6):1355-1381, 2006.

[12] M. B. Jones, C. P. Please, D. L. S McElwain, G. R. Fulford, A. P. Roberts, and M. J. Collins. Dynamics of tear film deposition and draining. Math. Med. Biol., 22(3):265-288, 2005. 
[13] T. A. Driscoll K. L. Maki, R. J. Braun and P. E. King-Smith. An overset grid method for the study of reflex tearing. Mathematical Medicine and Biology, doi:19.1093/immammb/dqn013, 2008.

[14] P. E. King-Smith, B. A. Fink, J. J. Nichols, K. K. Nichols, R. J. Braun, and G. B. McFadden. The contribution of lipid layer movement to tear film thinning and breakup. Invest. Ophthal. Vis. Sci., 50(6):2747-2756, 2009.

[15] R Krechetnikov and G. M. Homsy. Surfactant effects in the landau-levich problem. J. Fluid Mech., 559:429-450, 2006.

[16] M. A. Lemp. The definition and classification of dry eye disease. The Ocular Surface, 5(2):7592, 2007.

[17] E. A. MacDonald and D. M. Maurice. The kinetics of the tear fluid under the lower lid. Exp. Eye Res., 53(4):421-425, 1991.

[18] W. D. Mathers. Ocular evaporation in meibomian gland dysfunction and dry eye. Ophthalmology, 100(3):347-351, 1993.

[19] J. P. McCulley and W. Shine. A compositional based model for the tear film lipid layer. Trans. Am. Ophthal. Soc., 95:80-93, 1997.

[20] S. Mishima, A. Gasset, S. D. Klyce Jr., and J. L. Baum. Determination of tear volume and tear flow. Invest. Ophthal. and Vis. Sci., 5:264-276, 1966.

[21] A. Nagyova and J.M. Tiffany. Components responsible for the surface tension of the human tears. Curr. Eye Res., 19(1):4-11, 1999.

[22] H. Ockendon and J. R. Ockendon. Viscous flow. Cambridge University Press, Cambridge, 1995.

[23] E. K. Sakata and J. C. Berg. Surface diffusion in monolayers. Indust. and Eng. Chem. Fund., 8(3):570-575, 1969.

[24] H. A. Stone. A simple derivation of the time-dependent convective-diffusion equation for surfactant transport along a deforming interface. Physics of Fluids A, 2(1):111-112, 1990.

[25] J. M. Tiffany, N. Winter, and G. Bliss. Tear film stability and tear surface tension. Curr. Eye Res., 8:507-515, 1989.

[26] H. Wong, I. Fatt, and C.J. Radke. Deposition and thinning of the human tear film. J. Coll. Interf. Sci., 184(1):44-51, 1996. 



\section{RECENT REPORTS}

2009

01/09 A Mass and Solute Balance Model for Tear Volume and Osmolar- Gaffney ity in The Normal And The Dry Eye Tiffany

Yokoi

Bron

02/09 Diffusion and permeation in binary solutions Peppin

03/09 On the modelling of biological patterns with mechanochemical Moreo models: insights from analysis and computation Gaffney

Garcia-Aznar

Doblare

04/09 Stability analysis of reaction-diffusion systems with timem- Madzvamuse dependent coefficients on growing domains

Gaffney

Maini

05/09 Onsager reciprocity in premelting solids

Peppin

Spannuth

Wettlaufer

06/09 Inherent noise can facilitate coherence in collective swarm motion

Yates et al.

07/09 Solving the Coupled System Improves Computational Efficiency of the Bidomain Equations

Southern

Plank

Vigmond

Whiteley

08/09 Model reduction using a posteriori analysis

Whiteley

09/09 Equilibrium Order Parameters of Liquid Crystals in the LaudauDe Gennes Theory

10/09 Landau-De Gennes theory of nematic liquid crystals: the OseenFrank limit and beyond

Majumdar

Majumdar

Zarnescu

11/09 A Comparison of Numerical Methods used for Finite Element Modelling of Soft Tissue Deformation

Pathmanathan

Gavaghan

Whiteley

12/09 From Individual to Collective Behaviour of Unicellular Organisms: Recent Results and Open Problems

Xue

Othmer

Erban

13/09 Stochastic modelling of reaction-diffusion processes: algorithms Erban for bimolecular reactions

Chapman

14/09 Chaste: a test-driven approach to software development for phys-

Pitt-Francis et al. iological modelling 
15/09 Block triangular preconditioners for PDE constrained optimiza- Rees tion

Stoll

16/09 From microscopic to macroscopic descriptions of cell migration on

Baker growing domains

Yates

Erban

17/09 The Influence of Gene Expression Time Delays on GiererMeinhardt Pattern Formation Systems

Seirin Lee

Gaffney

Monk

18/09 Analysis of a stochastic chemical system close to a sniper bifurcation of its mean field model

Erban et al.

19/09 On the existence and the applications of modified equations for stochastic differential equations

20/09 Pebble bed: reflector treatment and pressure velocity coupling

21/09 A finite difference method for free boundary problems

Fornberg

22/09 Tangent unit-vector fields: nonabelian homotopy invariants and the Dirichlet energy

Majumdar

Robbins

Zyskin

23/09 Morphological instability of a nonequilibrium icecolloid interface

Peppin

Majumdar

Wettlaufer

Copies of these, and any other OCCAM reports can be obtained from:

Oxford Centre for Collaborative Applied Mathematics

Mathematical Institute

24 - 29 St Giles'

Oxford

OX1 3LB

England

www.maths.ox.ac.uk/occam 